\title{
Minimal Structures for the Implementation of Digital Rational Lossless Systems
}

\author{
ZINNUR DOĞANATA, MEMBER, IEEe, AND P. P. VAIDYANATHAN, SENIOR MEMBER, IEeE
}

\begin{abstract}
Digital lossless transfer matrices and vectors (power-complementary vectors) have recently found applications in digital filter bank systems, both single rate and multirate. In this paper, two new structures for the implementation of rational lossless systems are presented. The first structure represents a characterization of single-input, multioutput lossless systems in terms of complex planar rotations, whereas the second structure offers a representation of $\boldsymbol{M}$-input, $M$-output lossless systems in terms of unit-norm vectors. This property makes the second structure desirable in applications that involve optimization of the parameters. Modifications of the second structure for implementing single-input, multioutput, and LBR systems are also included. The main importance of the structures is that they are completely general, i.e., they span the entire set of $M \times 1$ and $M \times M$ lossless systems. This is demonstrated in the paper by showing that any such system can be synthesized using these structures. The structures are also minimal in the sense that they use the smallest number of scalar delays and parameters to implement a lossless system of given degree and dimensions. A design example to demonstrate the main results is included.
\end{abstract}

\section{INTRODUCTION}

$\mathrm{D}$ IGITAL filter banks [1]-[3], [7] find a variety of applications, as in subband coding systems [1], voiceprivacy systems [4], and spectral estimation systems [5]. A filter bank is basically a collection of $M$ filters which either splits a signal $x(n)$ into $M$ subband signals $x_{k}(n)$ (analysis bank, Fig. 1(a)) or combined $M$ signals $y_{k}(n)$ into one signal $y(n)$ (synthesis bank, Fig. 1(b)).

An analysis bank is said to be power complementary (PC) if the filters are such that

$$
\sum_{k=0}^{M-1}\left|H_{k}\left(e^{i \omega}\right)\right|^{2}=c \quad \forall \omega
$$

where $c$ is a nonzero constant. An analysis bank is characterized by an $M \times 1$ transfer matrix $\boldsymbol{h}(z)=\left(H_{0}(z)\right.$ $\left.H_{1}(z) \cdots H_{M-1}(z)\right)^{T}$ and a synthesis bank by a $1 \times M$ transfer matrix $f^{T}(z)=\left(F_{0}(z) F_{1}(z) \cdots F_{M-1}(z)\right)$. The PC property (1) of the analysis bank is equivalent to

$$
\boldsymbol{h}^{\dagger}\left(e^{i \omega}\right) \boldsymbol{h}\left(e^{i \omega}\right)=c \quad \forall \omega
$$

where the superscript dagger stands for transpose conjugation. This property has been called the "losslessness", property [6] in a number of past publications. If $\boldsymbol{h}(z)$ sat-

Manuscript received January 27, 1989; revised January 2, 1990. This work was supported in part by NSF Grants DCI 8552579 and MIP 8604456

Z. Doganata was with the Department of Electrical Engineering. California Institute of Technology. Pasadena, CA 91125. She is now with IBM T. J. Watson Research Center, Yorktown Heights, NY 10598.

P. P. Vaidyanathan is with the Department of Electrical Engineering. California Institute of Technology. Pasadena, CA 91125

IEEE Log Number 9038941

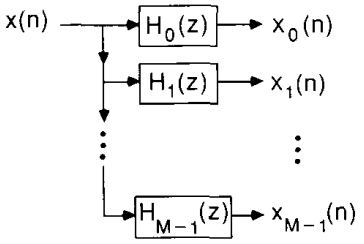

(a)

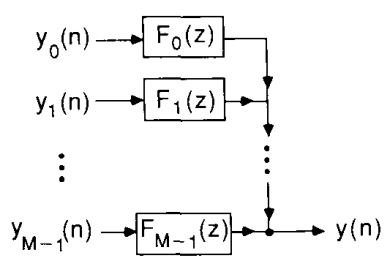

(b)

Fig. 1. (a) The analysis bank. (b) The synthesis bank.

isfies (2) we shall say that it is a lossless vector. The advantage of imposing this property has been discussed in [7], [8]. In certain applications, such as in multirate filter bank systems (called quadrature mirror filters or QMF), a more general type of losslessness property is often imposed. This is the losslessness of an $M \times M$ transfer matrix called the polyphase matrix, which has been elaborated in [7], [9].

Because of the importance of lossless vectors and matrices in filter bank systems, it is of interest to find general structures and realization procedures for such systems. Some partial results which deal with special cases have indeed been reported in the past. For example, [10] considers the synthesis of $M \times 1$ FIR lossless systems with real coefficients. On the other hand, [11], [12] deal with a special type of $2 \times 1$ IIR lossless system with real coefficients, where each of the IIR filters $H_{0}(z)$ and $H_{1}(z)$ is a linear combination of two all-pass functions. A recent tutorial on losslessness can be found in [27]. In [22], the problem of factorizing scattering matrices into degree-one factors is considered. Finally, [9] deals with a state-space approach for the realization of $M \times M$ real-coefficient FIR transfer matrices.

The purpose of this paper is to obtain a complete generalization of lossless systems, so that the results in [10][12] are special cases. Even though discrete-time lossless systems have been found to be of tremendous importance in the above-mentioned recent signal-processing applications, it has not been possible in the past to obtain a self- 
contained and unified documentation of discrete-time FIR and IIR lossless systems including $M \times M$ and $M \times 1$ systems. An excellent reference in continuous-time lossless systems is the text by Belevitch [15]. In this paper, we have chosen not to translate the results of [15] by use of bilinear transforms, for two reasons. First, a direct discrete-time approach leads to a self-contained presentation, opening up wider readership. And second, such a direct derivation is often simpler and leads to newer implementations. Some portions of the results of this paper have appeared in a recent conference proceedings [26].

This paper is organized as follows: In Section II, some interesting properties of lossless matrices are reviewed. In Section III, a general lattice structure for PC IIR vectors is derived and its synthesis procedure is described. A design example is also presented to demonstrate the results. In Section IV, a new characterization for degreeone IIR lossless matrices is introduced, and used to obtain structural representations for $M \times M$ lossless IIR matrices and $M \times 1$ PC IIR vectors of arbitrary degree. The $M \times$ $M$ structures derived in Section IV-B are generalized IIR versions of the FIR structures used earlier in [13] for QMF design, where it was necessary to optimize the coefficients of a lossless FIR real-coefficient polyphase matrix in order to obtain good analysis-bank filters which satisfy the perfect reconstruction requirement. The $M \times 1$ IIR structures derived in Section IV-C are again generalizations of an FIR version used in [14], once again for the QMF design problem. The $M \times 1$ synthesis procedure is useful in the process of initializing an analysis filter in [14], prior to the optimization process. Some interesting properties of these structures such as orthogonality and minimality are discussed in Section V. Finally, some concluding remarks are given in Section VI.

The following notations are used in the paper: Superscript $T$ stands for matrix (or vector) transposition, whereas the superscript dagger $(\dagger)$ stands for transposition followed by complex conjugation. Boldface letters indicate matrices and vectors. The superscript asterisk $(*)$ stands for complex conjugation, while the subscript asterisk denotes conjugation of coefficients of a function or a matrix. The tilde on a matrix $\boldsymbol{F}(z)$ is defined such that $\tilde{\boldsymbol{F}}(z)=\boldsymbol{F}_{*}^{T}\left(z^{-1}\right)$, and for matrices with real coefficients $\tilde{\boldsymbol{F}}(z)=\boldsymbol{F}^{T}\left(z^{-1}\right)$. Thus on the unit circle, $\tilde{\boldsymbol{F}}(z)=\boldsymbol{F}^{\dagger}(z)$. The hat accent on a polynomial $P(z)=\sum_{i=0}^{M-1} p_{i} z^{-i}$ is defined such that $\hat{P}(z)=z^{-(M-1)} P_{*}\left(z^{-1}\right)$. The notation $a(z) \mid b(z)$ will be read as $a(z)$ divides $b(z)$. Finally, the Euclidean norm of a vector $x$ is designated by the symbol $\|\boldsymbol{x}\|$, so that $\|\boldsymbol{x}\|=\sqrt{\boldsymbol{x}^{\leftarrow} \boldsymbol{x}}$.

\section{Multi-Input, Multioutput Lossless Systems: Some Definitions and Properties}

In this section, we will give the definitions and the mathematical background for multi-input multioutput (MIMO) lossless systems that will be required in the coming sections, as well as some properties of MIMO lossless systems that will not be put into use in this paper, but still are of considerable interest by themselves.

\section{A. Some Useful Definitions About MIMO Systems}

1) Matrix Fraction Descriptions for MIMO Systems: An $M$-input, $P$-output system characterized by a $P$ $\times M$ transfer matrix $\boldsymbol{H}(z)$ with rational entries can often be given a matrix fraction description (MFD) [17]. This description is an extension of the rational function representation for the scalar case. In the following, we will use the form

$$
\boldsymbol{H}(z)=\boldsymbol{Q}^{-1}(z) \boldsymbol{P}(z)
$$

known as a left MFD, where $Q(z)$ and $P(z)$, respectively, are the $P \times P$ denominator and $P \times M$ numerator matrices. The polynomial matrices $P(z)$ and $Q(z)$ can be expressed as

$$
\begin{aligned}
& \boldsymbol{P}(z)=\sum_{n=0}^{N} \boldsymbol{p}(n) z^{N-n} \\
& \boldsymbol{Q}(z)=\sum_{n=0}^{N} \boldsymbol{q}(n) z^{N-n}
\end{aligned}
$$

where $q(n)$ are $P \times P$ and $p(n)$ are $P \times M$. Notice that only positive powers of $z$ appear in (4). This is not a loss of generality since we can multiply the matrices $Q(z)$ and $\boldsymbol{P}(z)$ with the scalar $z^{-N}$ to obtain equivalent representations for $\boldsymbol{H}(z)$ in $z^{-1}$.

Given $\boldsymbol{P}(z)$ and $\boldsymbol{Q}(z)$, suppose that we can write

$$
\boldsymbol{Q}(z)=L(z) Q_{1}(z), \quad \boldsymbol{P}(z)=L(z) P_{1}(z)
$$

where $L(z)$ is a $P \times P$ polynomial matrix. Then $L(z)$ is said to be a left common divisor (LCD) of $\boldsymbol{Q}(z)$ and $\boldsymbol{P}(z)$. Note that $\boldsymbol{Q}_{1}^{-1}(z) \boldsymbol{P}_{1}(z)$ is also a valid MFD for $\boldsymbol{H}(z)$. An LCD $L(z)$ of $\boldsymbol{Q}(z)$ and $\boldsymbol{P}(z)$ is said to be a greatest LCD (GLCD) of $\boldsymbol{Q}(z)$ and $P(z)$ if every other LCD $L_{1}(z)$ of $\boldsymbol{Q}(z)$ and $\boldsymbol{P}(z)$ is a left-factor of $\boldsymbol{L}(z)$, i.e.,

$$
L(z)=L_{1}(z) W(z)
$$

for some polynomial matrix $\boldsymbol{W}(z)$. Given an MFD $\boldsymbol{Q}^{-1}(z)$ $\boldsymbol{P}(z)$ for $\boldsymbol{H}(z)$, if we can identify and cancel off a GLCD, the resulting MFD for $\boldsymbol{H}(z)$ is said to be irreducible. Note that irreducible MFD's are not unique, since given an irreducible MFD $Q^{-1}(z) P(z)$, we can generate infinitely many others of the form $Q^{-1}(z) \boldsymbol{P}(z)$ simply by writing

$$
Q_{1}(z)=W(z) Q(z), \quad P_{1}(z)=W(z) P(z)
$$

where $W(z)$ is any $P \times P$ unimodular matrix. The matrices $Q(z)$ and $\boldsymbol{P}(z)$ describing an irreducible MFD are said to be left coprime.

2) Poles and Zeros of a MIMO System: The transfer matrix $\boldsymbol{H}(z)$ is said to have a pole at $z_{p}$ if any of its entries has a pole ac $z_{p}$. If an irreducible MFD $Q^{-1}(z) \boldsymbol{P}(z)$ for $\boldsymbol{H}(z)$ is assumed, $z_{p}$ is a pole of $\boldsymbol{H}(z)$ if and only if it is a zero of the polynomial det $\boldsymbol{Q}(z)$.

The normal rank $r_{n}$ of $\boldsymbol{P}(z)$ is defined to be max - [ rank $\boldsymbol{P}(z)]$. We define $z_{0}$ to be zero of $\boldsymbol{H}(z)$, if rank $\boldsymbol{P}\left(z_{0}\right)<$

A square matrix $W(z)$ which has $\operatorname{det} W(z)=c$, where $c$ is a constant independent of $z$ is said to be unimodular 
$r_{n}$. For a system with $P=M=r_{n}$, the zeros $\boldsymbol{H}(z)$ coincide with the zeros of det $\boldsymbol{P}(z)$.

3) The Degree of a MIMO System: As in the scalar case, the smallest number of scalar delay elements $z^{-1}$ required to implement $\boldsymbol{H}(z)$ is called the degree (or McMillan degree) of $\boldsymbol{H}(z)$. To determine the degree of $\boldsymbol{H}(z)$, we start with an irreducible MFD $\boldsymbol{Q}^{-1}(z) \boldsymbol{P}(z)$ for $\boldsymbol{H}(z)$. It can be proved [17] that with such an MFD

$$
\operatorname{deg} \boldsymbol{H}(z)=\operatorname{deg} \operatorname{det} \boldsymbol{Q}(z) .
$$

It is meaningful to consider an irreducible MFD in order to define the degree, since if the MFD is reducible, one can cancel off LCD's to obtain other MDF's which have lower order denominator determinants. This process can be continued until an irreducible MFD for $\boldsymbol{H}(z)$ is reached.

It is in general not possible to determine the degree of a given matrix $\boldsymbol{H}(z)$ by inspection. However, in the special case of an $M \times 1$ vector

$$
\boldsymbol{H}(z)=\left(\begin{array}{llll}
P_{0}(z) & P_{1}(z) & \cdots & P_{M-1}(z)
\end{array}\right)^{T} / d(z)
$$

where the polynomials $P_{i}(z), 0 \leq i \leq M-1$ and $d(z)$ do not have any factors common to all of them, the degree is given by the maximum degree over all rational functions $P_{i}(z) / d(z)$.

\section{B. MIMO Lossless Systems}

A $P \times M$ transfer matrix $\boldsymbol{H}(z)$ with rational entries is lossless if all its entries are stable, and if it is unitary on the unit circle, i.e., it satisfies

$$
\boldsymbol{H}^{\dagger}\left(e^{i \omega}\right) \boldsymbol{H}\left(e^{i \omega}\right)=c \boldsymbol{I}_{M} \quad \forall \omega
$$

for some positive scalar $c$. Equation (10) can also be written as

$$
\tilde{\boldsymbol{H}}(z) \boldsymbol{H}(z)=c \boldsymbol{I}_{M} \quad \text { for } z=e^{i \omega} .
$$

Since (11) holds for every point on the unit circle, and $\boldsymbol{H}(z)$ and $\tilde{\boldsymbol{H}}(z)$ are analytic except for an isolated set of points in the $z$ plane, we conclude by analytical continuation [15] that it holds for all values of $z$. Hence we can also identify a matrix $\boldsymbol{H}(z)$ as lossless if it is stable and satisfies

$$
\tilde{\boldsymbol{H}}(z) \boldsymbol{H}(z) \doteq c \boldsymbol{I} \quad \forall z .
$$

A special case of lossless matrices are stable PC vectors. An $M \times 1$ vector $\boldsymbol{H}(z)=\left(H_{0}(z) H_{1}(z) \cdots\right.$ $\left.H_{M-1}(z)\right)^{T}$ is said to be PC if (1) holds. Another special case of lossless matrices are lossless bounded real (LBR) matrices. A lossless matrix $\boldsymbol{H}(z)$ is LBR if $\boldsymbol{H}(z)$ is real for real $z$.

A matrix $\boldsymbol{H}(z)$ which satisfies (11) for all $z$ is said to be paraunitary. Thus a lossless system is stable and paraunitary. The paraunitary property of a lossless $\boldsymbol{H}(z)$ induces several other secondary properties on $\boldsymbol{H}(z)$. In the following, we will state some of these properties without proof (the proofs can be found in [27]). Some of these properties will be crucially employed in the coming sections.
Property 1: The determinant of a lossless square ma$\operatorname{trix} H(z)$ is a stable all-pass function. In the special case where $\boldsymbol{H}(z)$ is FIR, the determinant is a pure delay.

Property 2: Given a square lossless matrix $\boldsymbol{H}(z), \alpha$ is a pole if and only if $1 / \alpha^{*}$ is a zero.

Property 3: For a square lossless matrix $\boldsymbol{H}(z)$, deg $\boldsymbol{H}(z)=\operatorname{deg} \operatorname{det} \boldsymbol{H}(z)$.

Property 3 is a very important characteristic of lossless matrices which will be used several times in the coming sections.

\section{The Smith-McMillan Form of an $M \times M$ Lossless Matrix}

In the following, we will focus on the Smith-McMillan form [15], [17] of an $M \times M$ lossless matrix $\boldsymbol{H}(z)$. This result is a discrete-time version of the one to be found in the classical text on network theory by Belevitch [15].

Let us first consider an $M \times M$ matrix $G(z)$ with rational entries in $z$ that are in reduced form. $\boldsymbol{G}(z)$ can be written as

$$
G(z)=\frac{N(z)}{d(z)}
$$

where $N(z)$ is an $M \times M$ polynomial matrix and $d(z)$ is the monic least common multiple of the denominators of the entries of $G(z)$. It can be shown [23] that $N(z)$ can be expressed as

$$
\boldsymbol{N}(z)=\boldsymbol{U}(z) \boldsymbol{\Lambda}(z) \boldsymbol{V}(z)
$$

where $U(z)$ and $V(z)$ are $M \times M$ unimodular matrices and $\Lambda(z)$ is the Smith form [17] of $N(z)$, given by

$$
\begin{aligned}
& \mathbf{\Lambda}(z)=\begin{array}{l}
r \\
M-r
\end{array}\left(\begin{array}{cc}
\operatorname{diag}\left[\lambda_{i}(z)\right] & \mathbf{0} \\
\mathbf{0} & \mathbf{0}
\end{array}\right), \\
& r \text { : normal rank of } N(z)
\end{aligned}
$$

with the polynomials $\lambda_{i}(z)$ satisfying the divisibility property

$$
\lambda_{i}(z) \mid \lambda_{i+1}(z), \quad 0 \leq i \leq r-2 .
$$

Let us now consider

$$
\boldsymbol{U}^{-1}(z) \boldsymbol{G}(z) \boldsymbol{V}^{-1}(z)=\frac{\boldsymbol{\Lambda}(z)}{d(z)}
$$

and reduce the entries of $\Lambda(z) / d(z)$ to the lowest terms, i.e., write

$$
\frac{\lambda_{i}(z)}{d(z)}=\frac{\epsilon_{i}(z)}{\phi_{i}(z)}, \quad 0 \leq i \leq r-1
$$

such that $\epsilon_{i}(z)$ and $\phi_{i}(z)$ are relatively prime. With this, $\boldsymbol{G}(z)$ can be expressed as

$$
\boldsymbol{G}(z)=\boldsymbol{U}(z) \boldsymbol{\Lambda}(z) \boldsymbol{V}(z)
$$

where the matrix $\boldsymbol{\Lambda}(z)$ given by

$$
\boldsymbol{\Lambda}(z)={ }_{M-r}\left(\begin{array}{cc}
\operatorname{diag} \mid \frac{\epsilon_{i}(z)}{\phi_{i}(z)} & \mathbf{0} \\
\mathbf{0} & \mathbf{0}
\end{array}\right)
$$


is known as the Smith-McMillan form [24] of $\boldsymbol{G}(z)$ and the polynomials $\epsilon_{i}(z), \phi_{i}(z)$ satisfy the obvious divisibility properties

$$
\begin{aligned}
& \epsilon_{i}(z) \mid \epsilon_{i+1}(z) \\
& \phi_{i+1}(z) \mid \phi_{i}(z)
\end{aligned}
$$

for $0 \leq i \leq r-2$. The poles and zeros of $G(z)$ can alternatively be defined as the roots of the denominator polynomials $\phi_{i}(z)$ and the numerator polynomials $\epsilon_{i}(z)$, respectively, of the Smith-McMillan form $\boldsymbol{\Lambda}(z)$. The polynomial matrices $U(z)$ and $V(z)$ in (16a) are highly nonunique [23], whereas the Smith-McMillan form $\boldsymbol{\Lambda}(z)$ of $\boldsymbol{G}(z)$ is unique except for ordering of entries and scale factors. This uniqueness property of the Smith-McMillan form will be evident later when we consider the concept of valuations [25].

Let us now consider the Smith-McMillan form of an $M$ $\times M$ FIR lossless matrix $\boldsymbol{H}(z)$. Since $\boldsymbol{H}(z)$ is FIR, it follows from Property 2 of Section II-B that

$$
\operatorname{det} \boldsymbol{H}(z)=c_{1} z^{-(K-1)}
$$

where $c_{1}$ is a nonzero complex constant, and $K-1$ is the McMillan degree of $\boldsymbol{H}(z)$. On the other hand, since $\boldsymbol{H}(z)$ $=\boldsymbol{U}(z) \boldsymbol{\Lambda}(z) \boldsymbol{V}(z)$

$$
\operatorname{det} \boldsymbol{H}(z)=c_{2} \operatorname{det} \boldsymbol{\Lambda}(z)
$$

where $c_{2}=\operatorname{det} \boldsymbol{U}(z)$ det $\boldsymbol{V}(z)$ is a complex constant. It follows from the diagonal nature of the Smith-McMillan form $\Lambda(z)$, and a comparison of (17) and (18) that

$$
\mathbf{\Lambda}(z)=\operatorname{diag}\left[z^{-n_{i}}\right]
$$

where $n_{i}$ are integers such that $0 \leq n_{0} \leq n_{1} \leq \cdots \leq$ $n_{M-1}$ and $\sum_{k=0}^{M-1} n_{k}=K-1$.

The next thing to consider is the case of an $M \times M$ lossless matrix with rational entries in $z$. Before we do so, however, we will look into the concept of valuations [17], [25] which will be useful later in obtaining the SmithMcMillan form of such a matrix. Suppose that we write a rational function $g(z)$ as $g(z)=(p(z) / q(z))(z-$ $\alpha)^{l \alpha}$ where $p(z)$ and $q(z)$ are relatively prime and not divisible by $(z-\alpha)$, and $\alpha$ is a finite pole or zero of $g(z)$. The integer $v_{\alpha}$ is called the valuation of $g(z)$ at $\alpha$. This definition can be generalized for rational matrices in the following way [25]. Given a matrix $G(z)$ with rational entries, the $i$ th valuation of $\boldsymbol{G}(z)$ at $\alpha$ is defined as

$$
v_{\alpha}^{(i)}(\boldsymbol{G}) \triangleq \min \left[v_{\alpha}\left(|\boldsymbol{G}|^{(i)}\right)\right]
$$

where $\alpha$ ranges over the set of all finite poles and zeros of $G(z)$ and the minimum is taken over all $i \times i$ minors $|\boldsymbol{G}|^{(i)}$ of $\boldsymbol{G}(z)$.

Now suppose that we write the nontrivial part of the Smith-McMillan form of $\boldsymbol{G}(z)$ as

$$
\boldsymbol{\Lambda}(z)=\prod_{\alpha} \boldsymbol{\Lambda}_{\alpha}(z)
$$

where $\alpha$ ranges over the set of poles and zeros of $\boldsymbol{G}(z)$, and $\boldsymbol{\Lambda}_{\alpha}(z)$ has the form

$$
\boldsymbol{\Lambda}_{\alpha}(z)=\begin{aligned}
& r \\
& M-r
\end{aligned}\left(\begin{array}{cc}
\operatorname{diag}\left[(z-\alpha)^{\sigma_{i}(\alpha)}\right] & \mathbf{0} \\
\mathbf{0} & \mathbf{0}
\end{array}\right) .
$$

In (2lb), the integers $\sigma_{i}(\alpha)$ have positive sign if $\alpha$ appears as a zero on the $i$ th diagonal entry of $\Lambda(z)$ and negative sign if it appears as a pole. As a consequence of the divisibility properties stated in (16c), (16d), $\sigma_{i}(\alpha)$ satisfy $\sigma_{0}(\alpha) \leq \sigma_{1}(\alpha) \leq \cdots \leq \sigma_{r-1}(\alpha)$. Due to the special form of $\boldsymbol{\Lambda}_{\alpha}(z)$, it follows that

$$
\begin{gathered}
\sigma_{0}(\alpha)=v_{\alpha}^{(1)}\left(\mathbf{\Lambda}_{\alpha}\right) \\
\sigma_{1}=v_{\alpha}^{(2)}\left(\boldsymbol{\Lambda}_{\alpha}\right)-v_{\alpha}^{(1)}\left(\boldsymbol{\Lambda}_{\alpha}\right) \\
\vdots \\
\sigma_{r-1}(\alpha)=v_{\alpha}^{(r)}\left(\boldsymbol{\Lambda}_{\alpha}\right)-v_{\alpha}^{(r-1)}\left(\boldsymbol{\Lambda}_{\alpha}\right)
\end{gathered}
$$

where $\alpha$ is a finite pole or zero of $\boldsymbol{G}(z)$. However, it can be shown [17] using (16a), unimodularity of the matrices $\boldsymbol{U}(z), \boldsymbol{V}(z)$ and the Cauchy-Binet theorem [17], [19] that

$$
v_{\alpha}^{(i)}\left(\boldsymbol{\Lambda}_{\alpha}\right)=v_{\alpha}^{(i)}(\mathbf{\Lambda})=v_{\alpha}^{(i)}(\boldsymbol{G}) .
$$

This gives us a direct way of constructing the SmithMcMillan form $\boldsymbol{\Lambda}(z)$ of $\boldsymbol{G}(z)$ based entirely on the valuations of $\boldsymbol{G}(z)$. The method also demonstrates the uniqueness of the Smith-McMillan form.

Let us now consider an $\boldsymbol{M} \times \boldsymbol{M}$ lossless matrix $\boldsymbol{H}(z)$ with rational entries in $z$ and write

$$
\boldsymbol{H}(z)=\boldsymbol{U}_{1}(z) \boldsymbol{\Lambda}_{1}(z) \boldsymbol{V}_{1}(z)
$$

where $\boldsymbol{U}_{1}(z)$ and $\boldsymbol{V}_{1}(z)$ are unimodular matrices and $\boldsymbol{\Lambda}_{1}(z)$ $=\operatorname{diag}\left[\epsilon_{i}(z) / \phi_{i}(z)\right]$ is the Smith-McMillan form of $\boldsymbol{H}(z)$. In (24), all the matrices have entries that are functions of $z$. Let us now rewrite $\boldsymbol{H}(z)$ as a function of $z^{-1}$ rather than $z$. This results in a new rational matrix $G\left(z^{-1}\right)$ such that

$$
\boldsymbol{H}(z)=\boldsymbol{G}\left(z^{-1}\right)
$$

The matrix $G\left(z^{-1}\right)$ can be written as

$$
\boldsymbol{G}\left(z^{-1}\right)=\boldsymbol{U}_{2}\left(z^{-1}\right) \boldsymbol{\Lambda}_{2}\left(z^{-1}\right) \boldsymbol{V}_{2}\left(z^{-1}\right)
$$

where the $U_{2}\left(z^{-1}\right)$ and $V_{2}\left(z^{-1}\right)$ are unimodular matrices in $z^{-1}$ and $\boldsymbol{\Lambda}_{2}\left(z^{-1}\right)=\operatorname{diag}\left[\psi_{i}\left(z^{-1}\right) / \eta_{i}\left(z^{-1}\right)\right]$. We should note here, however, that since $G\left(z^{-1}\right)$ has entries in $z^{-1}$ rather than in $z$, the matrix $\boldsymbol{\Lambda}_{2}\left(z^{-1}\right)$ does not necessarily reflect the behavior of $\boldsymbol{G}\left(z^{-1}\right)$ at $z=0$. Furthermore, $\boldsymbol{\Lambda}_{2}\left(z^{-1}\right)$ is not a regular Smith-McMillan form in the sense that the sum of the degrees of the denominator polynomials $\eta_{i}\left(z^{-1}\right)$ does not necessarily equal the degree of $\boldsymbol{G}\left(z^{-1}\right)$. It follows from (25) and the constructability of $\boldsymbol{\Lambda}_{1}(z)$ and $\boldsymbol{\Lambda}_{2}\left(z^{-1}\right)$ from the valuations of $\boldsymbol{H}(z)$ and $\boldsymbol{G}\left(z^{-1}\right)$, respectively, that

$$
\frac{\epsilon_{i}(z)}{\phi_{i}(z)}=c_{j_{i}} z^{d_{i}} \frac{\psi_{j_{i}}\left(z^{-1}\right)}{\eta_{j_{i}}\left(z^{-1}\right)}, \quad 0 \leq i \leq M-1
$$


where $c_{j}$ is a complex constant, $d_{j_{i}}$ is an integer and $j_{0}$, $\cdots, j_{M-1}$ represents a permutation of the integers 0 , $\cdots, M-1$. On the other hand, since $\boldsymbol{H}(z)$ is lossless

$$
\boldsymbol{H}(z)=\left[\tilde{\boldsymbol{G}}\left(z^{-1}\right)\right]^{-1} .
$$

If we substitute (24) and (26) for $\boldsymbol{H}(z)$ and $\boldsymbol{G}\left(z^{-1}\right)$ in (28), we obtain

$$
\boldsymbol{U}_{1}(z) \boldsymbol{\Lambda}_{1}(z) \boldsymbol{V}_{1}(z)=\boldsymbol{U}_{2, *}^{-r}(z) \Lambda_{2, *}^{-1}(z) \boldsymbol{V}_{2, *}^{-T}(z)
$$

Since $U_{1}(z), U_{2}(z), U_{2, *}^{-T}(z)$, and $\boldsymbol{V}_{2, *}^{-T}(z)$ are all unimodular matrices in $z, \boldsymbol{\Lambda}_{1}(z)$ and $\boldsymbol{\Lambda}_{2 *}^{-1}(z)$ must both be Smith-McMillan forms for the same matrix $\boldsymbol{H}(z)$. It follows from the uniqueness of the Smith-McMillan form that $\boldsymbol{\Lambda}_{1}(z)$ and $\boldsymbol{\Lambda}_{2, *}^{-1}(z)$ are the same except for scale factors, delays, and a possible relabeling of entries, i.e.,

$$
\frac{\epsilon_{j}(z)}{\phi_{j}(z)}=c_{k_{j}} z^{d_{k_{1}}} \frac{\eta_{k_{j . *}}(z)}{\psi_{k_{j . *}}(z)} .
$$

If we substitute for $\eta_{k_{j * *}}(z) / \psi_{k_{j . *}}(z)$ in (30) using (27), we obtain

$$
\frac{\epsilon_{j}(z)}{\phi_{j}(z)}=c_{l_{j}} z^{d_{l_{1}}} \frac{\hat{\phi}_{l_{j}}(z)}{\hat{\epsilon}_{l_{j}}(z)}
$$

where $c_{l_{j}}$ is a complex constant, $d_{l_{j}}$ is an integer and $l_{0}$, $\cdots, l_{M-1}$ is a permutation of the integers $0, \cdots M-$ 1. Since $\epsilon_{j}(z)$ and $\phi_{j}(z)$ are relatively prime, (31) implies that

$$
\epsilon_{j}(z)=a_{l_{j}} z^{b_{1}} \hat{\phi}_{l_{j}}(z)
$$

where $b_{l_{j}}$ is an integer and $a_{l_{j}}$ is complex. It is intuitively clear (and is proved in the Appendix) that (32) together with the divisibility properties stated in $(16 \mathrm{c}),(16 \mathrm{~d}) \mathrm{fix}$ the permutation $l_{0}, \cdots, l_{M-1}$ as $l_{j}=M-1-j$, for 0 $\leq j \leq M-1$. With this, (32) becomes

$$
\epsilon_{j}(z)=a_{M-1-j} z^{d_{M-1-1}} \hat{\phi}_{M-1-j}(z)
$$

and $\boldsymbol{\Lambda}_{1}(z)$ has the form

$$
\mathbf{\Lambda}_{1}(z)=\operatorname{diag}\left[a_{M-1-j} z^{b_{M-1},} \frac{\hat{\phi}_{M-1-j}(z)}{\phi_{j}(z)}\right] .
$$

Note that some properties of lossless matrices stated in Section II-B such as the all-pass nature of the determinant and the existence of a pole at $1 / \alpha^{*}$ for every zero at $\alpha$ (and vice versa), follow as corrollaries of this result.

\section{A General Structure for PC IIR Vectors}

In this section, we will introduce a completely general structure for implementing PC IIR vectors. This structure is a generalization of the structure described in [10] for real-coefficient PC FIR vectors. In the following, we will consider the 2-component case first and then generalize the results to $M$ components.

\section{A. A Lattice Structure for Two-Component PC IIR Vectors}

Consider a PC IIR vector of degree $K-1$ which can be written as

$$
\boldsymbol{H}_{K-1}(z)=\frac{\left(\begin{array}{c}
P_{K-1}(z) \\
Q_{K-1}(z)
\end{array}\right)}{d_{K-1}(z)}
$$

where

$$
\begin{aligned}
P_{K-1}(z) & =\sum_{i=0}^{K-1} p_{K-1, i} z^{-i} \\
Q_{K-1}(z) & =\sum_{i=0}^{K-1} q_{K-1, i} z^{-i} \\
d_{K-1}(z) & =\prod_{i=1}^{K-1}\left(1-z_{i} z^{-1}\right), \quad\left|z_{i}\right|<1
\end{aligned}
$$

The scalars $p_{K-1, i}, q_{K-1 . i}$, and $z_{i}$ are in general complex. We assume without loss of generality that $P_{K-1}(z)$, $Q_{K-1}(z)$, and $d_{K-1}(z)$ do not have a factor common to all of them, as such a factor can be determined and cancelled. Losslessness of $\boldsymbol{H}_{K-1}(z)$ implies that

$$
\begin{gathered}
\tilde{P}_{K-1}(z) P_{K-1}(z)+\tilde{Q}_{K-1}(z) Q_{K-1}(z) \\
=\tilde{d}_{K-1}(z) d_{K-1}(z) \quad \forall z
\end{gathered}
$$

or, equivalently, taking the complex conjugate of both sides of (35a)

$$
\begin{gathered}
P_{K-1}\left(\frac{1}{z^{*}}\right) P_{K-1}^{*}(z)+Q_{K-1}\left(\frac{1}{z^{*}}\right) Q_{K-1}^{*}(z) \\
=d_{K-1}\left(\frac{1}{z^{*}}\right) d_{K-1}^{*}(z) \quad \forall z .
\end{gathered}
$$

We shall use this property in the synthesis procedure.

Given $\boldsymbol{H}_{K-1}(z)$ as in (34a), we would like to generate a lower order system

$$
\boldsymbol{H}_{K-2}(z)=\frac{\left(\begin{array}{l}
P_{K-2}(z) \\
Q_{K-2}(z)
\end{array}\right)}{d_{K-2}(z)}
$$

such that it is lossless (i.e., PC and stable) and of degree $K-2$. Repeated application of this process then results in a structural realization for $\boldsymbol{H}_{K-1}(z)$. Each element of $\boldsymbol{H}_{K-2}(z)$ should be generated by a linear combination of the elements $\boldsymbol{H}_{K-1}(z)$. Consider the simplest possible linear combination $\left[\alpha P_{K-1}(z)+\beta Q_{K-1}(z)\right] / d_{K-1}(z)$. This has a lower degree if $\alpha$ and $\beta$ are chosen such that $\alpha P_{K-1}(z)+\beta Q_{K-1}(z)$ has a factor $\left(1-z_{1} z^{-1}\right)$ which can be cancelled with the denominator $d_{K-1}(z)$. An obvious choice for this is to let $\alpha=0, \beta=1$ if $Q_{K-1}\left(z_{1}\right)$ $=0$, and $\alpha=1, \beta=-\left(P_{K-1}\left(z_{1}\right) / Q_{K-1}\left(z_{1}\right)\right)$ otherwise. Thus we have generated one component of $\boldsymbol{H}_{K-2}(z)$, viz $\left(P_{K-2}(z) / d_{K-2}(z)\right.$ where $P_{K-2}(z)$ and 
$d_{K-2}(z)$ are polynomials of degree less than $K-1$ given where

by

$$
\begin{array}{r}
P_{K-2}(z)=\frac{\alpha P_{K-1}(z)+\beta Q_{K-1}(z)}{1-z_{1} z^{-1}} \\
d_{K-2}(z)=\frac{d_{K-1}(z)}{1-z_{1} z^{-1}} .
\end{array}
$$

We now need to find the other linear combination which would generate the second component $Q_{K-2}(z) / d_{K-2}(z)$ of $\boldsymbol{H}_{K-2}(z)$. The complete reduction can be expressed as

$$
\frac{\left(\begin{array}{l}
P_{K-2}(z) \\
Q_{K-2}(z)
\end{array}\right)}{d_{K-2}(z)}=\frac{\left(\begin{array}{ll}
\alpha & \beta \\
a(z) & b(z)
\end{array}\right)\left(\begin{array}{l}
P_{K-1}(z) \\
Q_{K-1}(z)
\end{array}\right)}{d_{K-1}(z)} .
$$

It remains to choose $a(z)$ and $b(z)$ such that $a(z)$ $P_{K-1}(z)+b(z) Q_{K-1}(z)$ has the factor $\left(1-z_{1} z^{-1}\right)$. In addition, we require the $2 \times 2$ matrix in (38) to be paraunitary so that the left-hand side in $(38)$, which is $\boldsymbol{H}_{K-2}(z)$, is PC. One obvious choice of $a(z), b(z)$ which makes the $2 \times 2$ matrix paraunitary is

$$
a(z)=-\beta^{*}, \quad b(z)=\alpha^{*} .
$$

With this,

$$
\begin{aligned}
a(z) & P_{K-1}(z)+b(z) Q_{K-1}(z) \\
= & -\beta^{*} P_{K-1}(z)+\alpha^{*} Q_{K-1}(z)
\end{aligned}
$$

becomes $-P_{K-1}(z)$ if $Q_{K-1}\left(z_{1}\right)=0$, and

$$
\frac{P_{K-1}^{*}\left(z_{1}\right) P_{K-1}(z)+Q_{K-1}^{*}\left(z_{1}\right) Q_{K-1}(z)}{Q_{K-1}^{*}\left(z_{1}\right)}
$$

otherwise. In either case, in view of (35b) and the fact that $d_{k-1}\left(z_{1}\right)=0$, the linear combination (40a) becomes zero at $1 / z_{1}^{*}$ rather than at $z_{1}$. We shall therefore define

$$
\begin{aligned}
& a(z)=-\beta^{*} \frac{1-z_{1} z^{-1}}{-z_{1}^{*}+z^{-1}} \\
& b(z)=\alpha^{*} \frac{1-z_{1} z^{-1}}{-z_{1}^{*}+z^{-1}}
\end{aligned}
$$

so that

$$
\frac{a(z) P_{K-1}(z)+b(z) Q_{K-1}(z)}{d_{K-1}(z)}=\frac{Q_{K-2}(z)}{d_{K-2}(z)}
$$

where $d_{K-2}(z)$ is the $K-2$ degree polynomial defined in (37) and $Q_{K-2}(z)$ is the $K-2$ (or lower) degree polynomial

$$
Q_{K-2}(z)=\frac{-\beta^{*} P_{K-1}(z)+\alpha^{*} Q_{K-1}(z)}{-z_{1}^{*}+z^{-1}}
$$

With the choice of (41), we can write

$$
\boldsymbol{H}_{K-2}(z)=\boldsymbol{T}_{1}(z) \boldsymbol{H}_{K-1}(z)
$$

$$
\boldsymbol{T}_{1}(z)=\left(\begin{array}{cc}
1 & 0 \\
0 & \frac{1-z_{1} z^{-1}}{-z_{1}^{*}+z^{-1}}
\end{array}\right)\left(\begin{array}{cc}
\alpha & \beta \\
-\beta^{*} & \alpha^{*}
\end{array}\right)
$$

is paraunitary. This ensures that $\boldsymbol{H}_{K-2}(z)$ is PC. Since the poles of $\boldsymbol{H}_{K-2}(z)$ are a subset of the poles of $\boldsymbol{H}_{K-1}(z)$, stability of $\boldsymbol{H}_{K-2}(z)$ is guaranteed so that $\boldsymbol{H}_{K-2}(z)$ is a 2 $\times 1$ lossless system of reduced degree.

It is convenient to obtain a normalized matrix $S_{1}(z)$ by scaling $\boldsymbol{T}_{1}(z)$, by multiplying with the scalar $c_{1}=$ $1 / \sqrt{|\alpha|^{2}+|\beta|^{2}}$ so that $\tilde{S}_{1}(z) S_{1}(z)=I$ for all $z$. We would then have

$S_{1}(z)=\left(\begin{array}{cc}1 & 0 \\ 0 & \frac{1-z_{1} z^{-1}}{-z_{1}^{*}+z^{-1}}\end{array}\right)\left(\begin{array}{cc}c_{1} & s_{1} e^{-i \theta_{1}} \\ -s_{1} e^{i \theta_{1}} & c_{1}\end{array}\right)$

where $s_{1}$ and $c_{1}$ are real numbers such that $c_{1}^{2}+s_{1}^{2}=1$ and $\theta_{1}$ is a real quantity. After such normalization, we finally arrive at

$$
\boldsymbol{H}_{K-1}(z)=\boldsymbol{W}_{1}(z) \boldsymbol{H}_{K-2}(z)
$$

where $\boldsymbol{W}_{1}(z)=S_{1}^{-1}(z)$ so that

$\boldsymbol{W}_{1}(z)=\left(\begin{array}{cc}c_{1} & -s_{1} e^{-i \theta_{1}} \\ s_{1} e^{i \theta_{1}} & c_{1}\end{array}\right)\left(\begin{array}{cc}1 & 0 \\ 0 & \frac{-z_{1}^{*}+z^{-1}}{1-z_{1} z^{-1}}\end{array}\right)$.

Since $\boldsymbol{W}_{1}(z)$ is paraunitary and stable (because $\left|z_{1}\right|<1$, $z_{1}$ being a pole of $\left.\boldsymbol{H}_{K-1}(z)\right)$, we note that $\boldsymbol{W}_{1}(z)$ is lossless. This gives us a realization for the lossless system $\boldsymbol{H}_{K-1}(z)$ in terms of the lower degree lossless system $\boldsymbol{H}_{K-2}(z)$ and the $2 \times 2$ degree-one lossless system $\boldsymbol{W}_{1}(z)$, as illustrated in Fig. 2.

We thus have established degree reduction by extracting the pole at $z_{1}$. Clearly, this step can be repeated to extract the poles at $z_{j}, 2 \leq j \leq K-1$, resulting in a reduced degree $\mathrm{PC}$ vector each time, until finally a zerodegree PC (i.e., unit-norm) vector is reached. This can be expressed by the recursion

$$
\boldsymbol{H}_{K-1-j}(z)=\boldsymbol{S}_{j}(z) \boldsymbol{H}_{K-j}, 1 \leq j \leq K-1
$$

where $S_{j}(z)$ has the form

$$
S_{j}(z)=\left(\begin{array}{cc}
1 & 0 \\
0 & \frac{1-z_{j} z^{-1}}{-z_{j}^{*}+z^{-1}}
\end{array}\right)\left(\begin{array}{cc}
c_{j} & s_{j} e^{-i \theta_{j}} \\
-s_{j} e^{i \theta_{j}} & c_{j}
\end{array}\right)
$$

and $\boldsymbol{H}_{0}(z)=\boldsymbol{H}_{0}$ is a unit-norm constant vector. The complete synthesis procedure can be expressed as

$$
\boldsymbol{H}_{0}=\boldsymbol{S}_{K-1}(z) \cdots S_{2}(z) \boldsymbol{S}_{1}(z) \boldsymbol{H}_{K-1}(z) .
$$




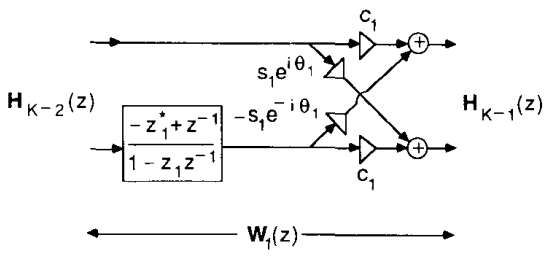

Fig. 2. Pertaining to the synthesis procedure of Section III-A.

\section{Defining}

$$
\begin{aligned}
\boldsymbol{W}_{j}(z)= & \boldsymbol{S}_{j}^{-1}(z)=\left(\begin{array}{ll}
c_{j} & -s_{j} e^{-i \theta_{j}} \\
s_{j} e^{i \theta_{j}} & c_{j}
\end{array}\right) \\
& \cdot\left(\begin{array}{cc}
1 & 0 \\
0 & \frac{-z_{j}^{*}+z^{-1}}{1-z_{j} z^{-1}}
\end{array}\right), \quad 1 \leq j \leq K-1
\end{aligned}
$$

we can write $\boldsymbol{H}_{K-1}(z)$ as

$$
\boldsymbol{H}_{K-1}(z)=\boldsymbol{W}_{1}(z) \cdots \boldsymbol{W}_{K-2}(z) \boldsymbol{W}_{K-1}(z) \boldsymbol{H}_{0} .
$$

Fig. 3 shows the implementation of this realization of $\boldsymbol{H}_{K-1}(z)$. The internal details of $\boldsymbol{W}_{k}(z)$ are as shown in Fig. 2 with 1 replaced by $k$. This gives us a procedure for synthesizing an arbitrary two-component PC IIR vector of degree $K-1$ as a cascade of $K-1$ lossless systems of degree one, terminated on the left by a constant $2 \times 1$ vector $\boldsymbol{H}_{0}$.

\section{B. Extension of the Synthesis Procedure to M-Component PC IIR Vectors}

The synthesis procedure described in Section III-A can be generalized to $M$-component PC IIR vectors of the form

$$
\begin{aligned}
\boldsymbol{H}_{K-1}(z)= & \left(P_{K-1}^{(0)}(z)\right. \\
& \left.P_{K-1}^{(1)}(z) \cdots P_{K-1}^{(M-1)}(z)\right)^{T} / d_{K-}
\end{aligned}
$$

where

$$
\begin{aligned}
P_{K-1}^{(k)}(z) & =\sum_{i=0}^{K-1} p_{K-1 . i}^{(k)} z^{-i}, \quad 0 \leq k \leq M-1 \\
d_{K-1}(z) & =\prod_{i=1}^{K-1}\left(1-z_{i} z^{-1}\right)
\end{aligned}
$$

Again, without loss of generality, it will be assumed that $P_{K-1}^{(k)}(z), 0 \leq k \leq M-1$ and $d_{K-1}(z)$ do not have any common factors. At a zero $z_{l}$ of $d_{K-1}(z)$, the polynomials $P_{K-1}^{(k)}(z)$ satisfy the generalized form of property $(35 \mathrm{~b})$ of Section III-A which is

$$
\sum_{k=0}^{M-1} P_{K-1}^{(k)}\left(\frac{1}{z_{l}^{*}}\right)\left[P_{K-1}^{(k)}\left(z_{l}\right)\right]^{*}=0
$$

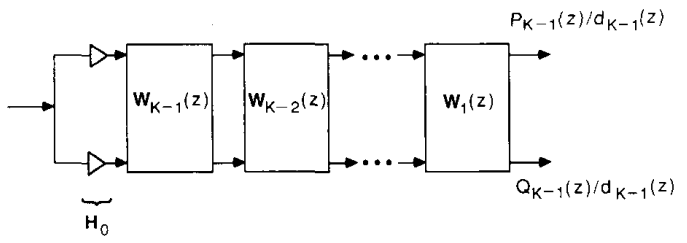

Fig. 3. The lattice structure implementation of a two-component PC IIR lossless vector $\boldsymbol{H}_{k-1}(z)$.

Before we give the generalized synthesis procedure, let us recall from Section III-A that given two polynomials $A_{0}(z)$ and $A_{1}(z)$, we can generate two new polynomials $B_{0}(z)$ and $B_{1}(z)$ such that $B_{0}(z)$ is zero at some point $z_{k}$, simply by writing

$$
\left(\begin{array}{l}
B_{0}(z) \\
B_{1}(z)
\end{array}\right)=\left(\begin{array}{cc}
c & s e^{-i \theta} \\
-s e^{i \theta} & c
\end{array}\right)\left(\begin{array}{l}
A_{0}(z) \\
A_{1}(z)
\end{array}\right)
$$

where

$$
\begin{aligned}
c & =\frac{\left|A_{1}\left(z_{k}\right)\right|}{\sqrt{\left|A_{0}\left(z_{k}\right)\right|^{2}+\left|A_{1}\left(z_{k}\right)\right|^{2}}} \\
s e^{-i \epsilon} & =\frac{-A_{0}\left(z_{k}\right) e^{-i a r g\left|A_{1}\left(z_{k}\right)\right|}}{\sqrt{\left|A_{0}\left(z_{k}\right)\right|^{2}+\left|A_{1}\left(z_{k}\right)\right|^{2}}}
\end{aligned}
$$

provided that $A_{0}(z)$ and $A_{1}(z)$ are not both zero at $z_{k}$. If that is the case, we can just let $c=1$ and $s=0$ in (52a).

Let us now consider two sets of polynomials $P_{K-1}^{(j)}(z)$ and $Q_{K-1}^{(j)}(z), 0 \leq j \leq M-1$, related by

$$
\begin{aligned}
\left(Q_{K-1}^{(0)}(z) \cdots Q_{K-1}^{(M-1)}(z)\right)^{T} & \\
= & U_{M-2, M-1} \cdots U_{1.2} U_{0,1}\left(P_{K-1}^{(0)}(z) \cdots\right. \\
& \left.P_{K-1}^{(M-1)}(z)\right)^{T}
\end{aligned}
$$

where the $M \times M$ matrix $U_{k, k+1}$ has the form

$$
\begin{gathered}
\boldsymbol{U}_{k, k+1}=\begin{array}{c}
k \\
k+1 \\
k+1
\end{array}\left(\begin{array}{cccl}
\boldsymbol{I}_{k} & \mathbf{0} & \mathbf{0} & \mathbf{0} \\
\mathbf{0} & c_{k} & s_{k} e^{-i \theta_{k}} & \mathbf{0} \\
\mathbf{0} & -s_{k} e^{i \theta_{k}} & c_{k} & \mathbf{0} \\
\mathbf{0} & \mathbf{0} & \mathbf{0} & \boldsymbol{I}_{M-k-2}
\end{array}\right) \\
0 \leq k \leq M-2 .
\end{gathered}
$$

It is evident from (53b) that the $k$ th and $(k+1)$ th outputs of $U_{k, k+1}$ are linear combinations of the respective input polynomials, and the other outputs are directly passed from the input in the order they originally appear. In (53a), let $\boldsymbol{U}_{k, k+1}$ be determined such that its $k$ th output polynomial has a zero at $z_{1}$. Clearly, $Q_{K-1}^{(k)}(z), 0 \leq k \leq M-$ 2 , can be made equal to zero at $z_{1}$ by determining $\boldsymbol{U}_{k, k+1}$ as described, for $0 \leq k \leq M-2$. Since $U_{k, k+1}$ are unitary matrices and $\boldsymbol{H}_{K-1}(z)$ is PC, the vector $\left(Q_{K-1}^{(0)}(z)\right.$ $\left.\cdots Q_{K-1}^{(M-1)}(z)\right)^{T} / d_{K-1}(z)$ is also PC. Therefore, at $z_{1}$, 
the polynomials $Q_{K-1}^{(k)}(z)$ satisfy

$$
\sum_{k=0}^{M-1} Q_{K-1}^{(k)}\left(\frac{1}{z_{1}^{*}}\right)\left[Q_{K-1}^{(k)}\left(z_{1}\right)\right]^{*}=0 .
$$

If we substitute $Q_{K-1}^{(k)}\left(z_{1}\right)=0$ for $0 \leq k \leq M-2$ in (54), we obtain

$$
Q_{K-1}^{(M-1)}\left(\frac{1}{z_{1}^{*}}\right)\left[Q_{K-1}^{(M-1)}\left(z_{1}\right)\right]^{*}=0
$$

which means that the $(M-1)$ th polynomial $Q_{K-1}^{(M-1)}(z)$ has a zero either at $z_{1}$ or at $1 / z_{1}^{*}$. Suppose that $Q_{K-1}^{(M-1)}\left(z_{1}\right)$ $=0$. Then all $Q_{K-1}^{(k)}(z)$, and therefore, all $P_{K-1}^{(k)}(z)$ have a zero at $z_{1}$. This, however, cannot be true since $P_{K-1}^{(k)}(z)$ and $d_{K-1}(z)$ do not have a factor common to all, by assumption. Therefore, (55) can only imply $Q_{K-1}^{(M-1)}\left(1 / z_{1}^{*}\right)=0$ so that we can write

$$
\begin{aligned}
\boldsymbol{H}_{K-2}(z)= & \left(\begin{array}{cc}
\boldsymbol{I}_{M-1} & 0 \\
0 & \frac{1-z_{1} z^{-1}}{-z_{1}^{*}+z^{-1}}
\end{array}\right) \\
& \cdot \boldsymbol{U}_{M-2, M-1} \cdots \boldsymbol{U}_{1,2} \boldsymbol{U}_{0.1} \boldsymbol{H}_{K-1}(z)
\end{aligned}
$$

where

$$
\boldsymbol{H}_{K-2}(z)=\left(P_{K-2}^{(0)}(z) \cdots P_{K-2}^{(M-1)}(z)\right)^{T} / d_{K-2}(z)
$$

and

$$
\begin{aligned}
P_{K-2}^{(j)}(z) & =\frac{Q_{K-1}^{(j)}(z)}{1-z_{1} z^{-1}}, \quad 0 \leq j \leq M-2 \\
P_{K-2}^{(M-1)}(z) & =\frac{Q_{K-1}^{(M-1)}(z)}{-z_{1}^{*}+z^{-1}} \\
d_{K-2}(z) & =\frac{d_{K-1}(z)}{1-z_{1} z^{-1}} .
\end{aligned}
$$

We have thus obtained an IIR PC vector $\boldsymbol{H}_{K-2}(z)$ of degree $K-2$ from $\boldsymbol{H}_{K-1}(z)$ by extracting its pole at $z_{1}$. Clearly we can repeat the described step to extract the other poles. If we define

$$
\begin{aligned}
\boldsymbol{S}_{j}(z)= & \left(\begin{array}{cc}
\boldsymbol{I}_{M-1} & \mathbf{0} \\
\mathbf{0} & \frac{1-z_{j} z^{-1}}{-z_{j}^{*}+z^{-1}}
\end{array}\right) \\
& \cdot \boldsymbol{U}_{M-2 . M-1}^{(j)} \cdots \boldsymbol{U}_{\mathrm{I} .2}^{(j)} \boldsymbol{U}_{0.1}^{(j)}
\end{aligned}
$$

(where the superscript $j$ is a reminder that we are working with the $j$ th pole $z_{j}$ ), we can describe this process by the recursion

$$
\boldsymbol{H}_{K-1-j}(z)=\boldsymbol{S}_{j}(z) \boldsymbol{H}_{K-j}(z), \quad 1 \leq j \leq K-1
$$

where the degree of the resultant IIR PC vector reduces by one at each step until finally a zero-degree unit-norm vector $\boldsymbol{H}_{0}$ is reached. Thus we can express $\boldsymbol{H}_{K-1}(z)$ as

$$
\boldsymbol{H}_{K-1}(z)=\boldsymbol{W}_{1}(z) \boldsymbol{W}_{2}(z) \cdots \boldsymbol{W}_{K-1}(z) \boldsymbol{H}_{0}
$$

where

$$
\begin{aligned}
\boldsymbol{W}_{j}(z)= & \boldsymbol{S}_{j}^{-1}(z)=\left[\boldsymbol{U}_{0.1}^{(j)}\right]^{\dagger}\left[\boldsymbol{U}_{1.2}^{(j)}\right]^{\dagger} \\
& \cdots\left[\boldsymbol{U}_{M-2, M-1}^{(j)}\right]^{\dagger}\left(\begin{array}{cc}
\boldsymbol{I}_{M-1} & \mathbf{0} \\
\mathbf{0} & \frac{-z_{j}^{*}+z^{-1}}{1-z_{j} z^{-1}}
\end{array}\right) .
\end{aligned}
$$

This expression results in the complete lattice structure implementation for $\boldsymbol{H}_{K-1}(z)$ shown in Fig. 4.

Before we conclude this section, we will briefly describe an exercise that we carried out to demonstrate that the synthesis procedure of this section really works. Two fifth-order elliptic filters $H_{0}(z)=N_{0}(z) / D_{0}(z)$ and $H_{2}(z)$ $=N_{2}(z) / D_{2}(z)$ were designed independently and then scaled such that $\left|H_{0}\left(e^{i \omega}\right)\right|^{2}+\left|H_{2}\left(e^{i \omega}\right)\right|^{2} \leq 1, \forall \omega$. A third filter $H_{1}(z)=N_{1}(z) / D_{1}(z)$ was designed with $D_{1}(z)$ $=D_{0}(z) D_{2}(z)$ and

$$
\begin{aligned}
\tilde{N}_{1}(z) N_{1}(z)= & \tilde{D}_{0}(z) D_{0}(z) \tilde{D}_{2}(z) D_{2}(z) \\
& -\tilde{N}_{0}(z) N_{0}(z) \tilde{D}_{2}(z) D_{2}(z) \\
& -\tilde{N}_{2}(z) N_{2}(z) \tilde{D}_{0}(z) D_{0}(z)
\end{aligned}
$$

so that the vector $\boldsymbol{H}(z)=\left(H_{0}(z) H_{1}(z) H_{2}(z)\right)^{T}$ is PC. $\boldsymbol{H}(z)$ was then synthesized using the procedure described above. The lattice coefficients obtained as a result of the synthesis process were used to reconstruct the three filters. The magnitude response plots of the reconstructed filters are shown in Fig. 5 and agree completely with the responses of the original filters. This example confirms that the synthesis procedure of this section can be used to synthesize a given PC IIR vector as the cascaded lattice structure shown in Fig. 4.

\section{The Minimality of the Structure}

The structure of Fig. 4 uses $K-1$ scalar delay elements to implement a PC IIR vector of degree $K-1$, hence it is minimal. In the following, we will show that it is also minimal in the sense that it uses the smallest possible number of parameters required to implement a completely general PC IIR vector of given degree and dimension.

Consider an $M \times 1 \mathrm{PC}$ IIR vector $\boldsymbol{H}_{K-1}(z)$ as given by (50). We will calculate the degrees of freedom that $\boldsymbol{H}_{K-1}(z)$ has. Note that $\boldsymbol{H}_{K-1}(z)$ satisfies the paraunitary condition

$$
\sum_{j=0}^{M-1} \tilde{P}_{K-1}^{(j)}(z) P_{K-1}^{(j)}(z)=\tilde{d}_{K-1}(z) d_{K-1}(z) .
$$

Both sides of $(60)$ are polynomials of order $2(K-1)$ displaying complex conjugate coefficient symmetry. If the coefficients of like terms on both sides are equated, we obtain $K$ non-redundant equalities, $K-1$ of which are 


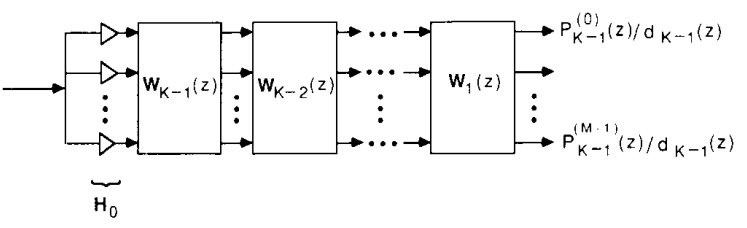

(a)

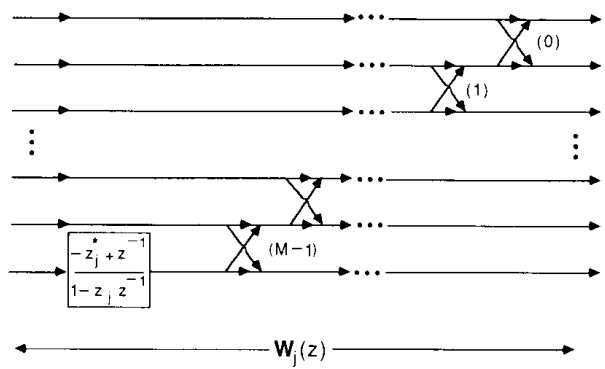

(b)

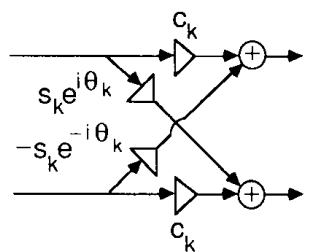

(c)

Fig. 4. (a) The lattice structure implementation of an $M$-component IIR lossless vector $\boldsymbol{H}_{k-1}(z)$. (b) Internal details of $\boldsymbol{W}_{j}(z)$. (c) Internal details of the $k$ th crisscross in $W_{j}(z)$

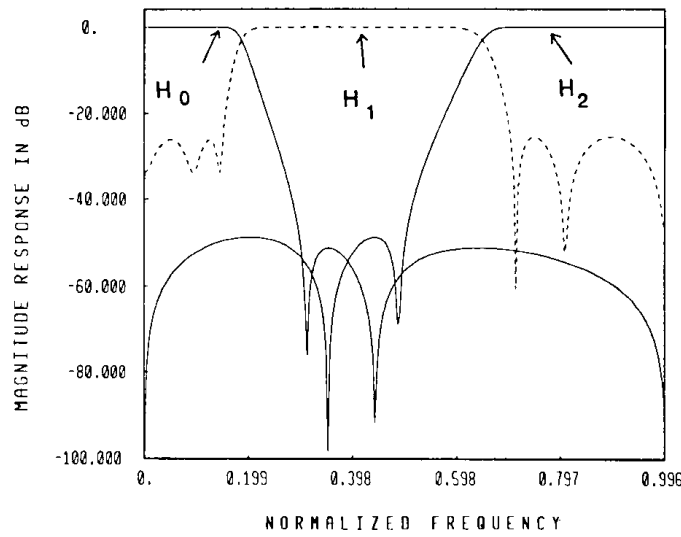

Fig. 5. Magnitude response plots of $\boldsymbol{H}_{0}(z), \boldsymbol{H}_{1}(z)$, and $\boldsymbol{H}_{2}(z)$.

complex, i.e., equivalent to two equations. Therefore, the total number of constraints is $2 K-1$. On the other hand, $\boldsymbol{H}_{K-1}(z)$ has $2 M K+2(K-1)$ unknowns which are the (complex) coefficients and poles. Subtracting the number of constraints from the number of unknowns, we find that $\boldsymbol{H}_{K-1}(z)$ has a total if $2 M K-1$ degrees of freedom.

Let us now suppose that we implement $\boldsymbol{H}_{K-1}(z)$ using the structure of Section III-B. It can easily be shown that this implementation has exactly $2 M K-1$ parameters.
Hence the structure of Section III-B represents a general $M \times 1$ PC IIR vector of degree $K-1$, using as many parameters as the number of degrees of freedom that such a vector has. Therefore the structure of Section III-B is minimal in the number of parameters it uses.

\section{Structures for $M \times M$ IIR Lossless Systems}

In this section, we consider some structural representations for $M \times M$ and $M \times 1$ lossless systems based on an algebraic form for general, degree-one IIR lossless matrices.

\section{A. A General Form for $M \times M$ Degree-One IIR Lossless Matrices}

Let us consider an $M \times M$ degree-one lossless transfer matrix $\boldsymbol{H}_{1}(z)$ with FIR entries. Using the fact that $\boldsymbol{H}_{1}(z)$ is unitary at all frequencies $\omega_{0}$ on the unit circle, we can express $\boldsymbol{H}_{1}(z)$ as

$$
\boldsymbol{H}_{1}(z)=\left(1-e^{i \omega \mid} z^{-1}\right) \boldsymbol{S}+\boldsymbol{R}
$$

where $S$ is $M \times M$, and $R$ is $M \times M$ unitary. It is worthwhile noting here that for a given FIR transfer matrix $\boldsymbol{G}(z)=\boldsymbol{g}_{0}+z^{-1} \boldsymbol{g}_{1}$, where $\boldsymbol{g}_{1} \neq 0$, deg $\boldsymbol{G}(z) \geq 1$. It can furthermore be proved that $\operatorname{deg} \boldsymbol{G}(z)=1$ if and only if rank $g_{1}=1$. Thus the form (61) in general represents matrices with degree equal to at least unity, the condition for equality being that $S$ is of unit rank.

Applying the paraunitary condition to (61), and working out the details (which can be found in [27]), we obtain the following result:

Theorem: An $M \times M$ matrix is lossless of degree-one if and only if it can be expressed as

$$
\left[\boldsymbol{I}-\boldsymbol{v} \boldsymbol{v}^{\dagger}+z^{-1} e^{i \omega_{0}} \boldsymbol{v} \boldsymbol{v}^{\dagger}\right] \boldsymbol{R}
$$

where $v$ is an $M \times 1$ constant unit-norm vector, $\boldsymbol{R}$ is an $M \times M$ constant unitary matrix, and $e^{i \omega()}$ is a real number.

Let us now consider an $M \times M$ lossless matrix of degree-one with rational entries. Such a matrix can be represented by the general form

$$
\boldsymbol{H}_{1}(z)=\frac{\boldsymbol{h}_{0}+z^{-1} \boldsymbol{h}_{1}}{1-a z^{-1}}
$$

where $\boldsymbol{h}_{0}$ and $\boldsymbol{h}_{1}$ are $\boldsymbol{M} \times \boldsymbol{M}$ constant matrices with complex-valued entries, and $a$ is a complex scalar that represents the pole of the system. Since $H_{1}(z)$ is stable, $|a|$ $<1$.

It can easily be verified that $\boldsymbol{H}_{1}(z)$ can also be represented as

$$
\boldsymbol{H}_{1}(z)=\frac{-a^{*}+z^{-1}}{1-a z^{-1}} e^{i \omega \mid} \boldsymbol{U}+\boldsymbol{V}
$$

where $\boldsymbol{U}$ and $\boldsymbol{V}$ are $M \times M$ constant matrices with complex entries. If we apply the paraunitary condition to (64), we obtain (after simplifications and collecting of like 
powers of $z$ ), the following conditions:

$$
\begin{aligned}
& \left(1+|a|^{2}\right) \boldsymbol{V} \boldsymbol{V}^{\dagger}-2 a^{*} e^{i \omega_{0}} \boldsymbol{U} \boldsymbol{V}^{\dagger}+\left(1+|a|^{2}\right) \boldsymbol{U} \boldsymbol{U}^{2} \\
& =\left(1+|a|^{2}\right) \boldsymbol{I} \\
& -a \boldsymbol{V} \boldsymbol{V}^{\dagger}+a^{2} e^{-i \omega 0} \boldsymbol{V} \boldsymbol{U}^{\dagger}+e^{i \omega 0} \boldsymbol{U} \boldsymbol{V}^{\dagger}-a \boldsymbol{U} \boldsymbol{U}^{\dagger}=-a \boldsymbol{I} \\
& -a^{*} \boldsymbol{V} \boldsymbol{V}^{\dagger}+e^{-i \omega 0} \boldsymbol{V} \boldsymbol{U}^{\dagger}+a^{* 2} e^{i \omega 0} \boldsymbol{U} \boldsymbol{V}^{\dagger}-a^{*} \boldsymbol{U} \boldsymbol{U}^{\dagger} \\
& =-a^{*} \boldsymbol{I} .
\end{aligned}
$$

Note that if we take the + of both sides of $(65 \mathrm{c})$, we obtain $(65 b)$. Therefore, we will consider only (65a) and (65b) as necessary conditions. Now if we scale (65a) by $1 /\left(1+|a|^{2}\right)$, and $(65 b)$ by $-(1 / a)$, we obtain

$$
\begin{gathered}
\boldsymbol{V} \boldsymbol{V}^{\dagger}-\frac{2 a e^{-i \omega_{0}} \boldsymbol{V} \boldsymbol{U}^{\dagger}+2 a^{*} e^{i \omega_{0}} \boldsymbol{U} \boldsymbol{V}^{\dagger}}{1+|a|^{2}}+\boldsymbol{U} \boldsymbol{U}^{\dagger}=\boldsymbol{I} \\
\boldsymbol{V} \boldsymbol{V}^{\dagger}-a e^{-i \omega_{0}} \boldsymbol{V} \boldsymbol{U}^{\dagger}-\frac{1}{a} e^{i \omega_{0}} \boldsymbol{U} \boldsymbol{V}^{\dagger}+\boldsymbol{U} \boldsymbol{U}^{\dagger}=\boldsymbol{I} .
\end{gathered}
$$

Subtracting (66b) from (66a), and simplifying yield

$$
\frac{1-|a|^{2}}{1+|a|^{2}}\left(-a e^{-i \omega_{0}} \boldsymbol{V} \boldsymbol{U}^{\dagger}+\frac{1}{a} e^{i \omega_{0}} \boldsymbol{U} \boldsymbol{V}^{\dagger}\right)=\mathbf{0}
$$

Since $|a| \neq 1$, the term inside the parenthesis in (67) must be $\mathbf{0}$, i.e., we must have

$$
\boldsymbol{V} \boldsymbol{U}^{\dagger}=\frac{1}{a^{2}} e^{i 2 \omega_{0}} \boldsymbol{U} \boldsymbol{V}^{\dagger}
$$

If we take the $\dagger$ of both sides of (68), and substitute the expression thus found for $\boldsymbol{U} \boldsymbol{V}^{\dagger}$ back into (68), we obtain

$$
\boldsymbol{V} \boldsymbol{U}^{\dagger}=\frac{1}{|a|^{4}} \boldsymbol{V} \boldsymbol{U}^{\dagger}
$$

which can only be satisfied if $|a|^{4}=1$ or $\boldsymbol{V} \boldsymbol{U}^{\dagger}=\mathbf{0}$. Since $|a|<1$ for stability reasons, (69) implies that $V U^{\dagger}=$ $\boldsymbol{U} \boldsymbol{V}^{\dagger}=\mathbf{0}$. Now this result can be substituted in (66a) and (66b) to get a simpler set of necessary conditions which is

$$
\begin{aligned}
\boldsymbol{V} \boldsymbol{V}^{\dagger}+\boldsymbol{U} \boldsymbol{U}^{\dagger} & =\boldsymbol{I}_{M} \\
\boldsymbol{V} \boldsymbol{U}^{\dagger} & =\mathbf{0} .
\end{aligned}
$$

But (70) is exactly the set of conditions that we would obtain if we imposed paraunitariness on the FIR form $V$ $+z^{-1} e^{i \omega_{0}} U$ where $V$ and $U$ are constant $M \times M$ matrices. This result enables us to obtain a general form for degreeone IIR lossless matrices simply by substituting $\left(-a^{*}+\right.$ $\left.z^{-1}\right) /\left(1-a z^{-1}\right)$ for $z^{-1}$ in the general form for degreeone FIR lossless matrices given by (62). Thus an $M \times M$ IIR lossless matrix $\boldsymbol{H}_{1}(z)$ of degree-one can always be written as

$$
\boldsymbol{H}_{1}(z)=\left[\boldsymbol{I}-\boldsymbol{v} \boldsymbol{v}^{\dagger}+e^{i \omega_{0}} \frac{-a^{*}+z^{-1}}{1-a z^{-1}} \boldsymbol{v} \boldsymbol{v}^{\dagger}\right] \boldsymbol{R}
$$

where $v$ is an $M \times 1$ unit-norm, complex-valued vector, $\boldsymbol{R}$ is an $M \times M$ unitary matrix, $0 \leq \omega_{0}<2 \pi$ and $|a|<$ 1. The converse statement that (71) indeed represents a lossless matrix follows since it was obtained by a lossless transformation from the lossless FIR form (62) [18]

\section{B. A General Form for $M \times M$ IIR Lossless Matrices} with Arbitrary Degree

Consider the product

$$
V_{1}(z) V_{2}(z) \cdots V_{K-1}(z)
$$

where

$$
\begin{gathered}
\boldsymbol{V}_{i}(z)=\boldsymbol{I}-\boldsymbol{v}_{i} \boldsymbol{v}_{i}^{\dagger}+\frac{-a_{i}^{*}+z^{-1}}{1-a_{i} z^{-1}} \boldsymbol{v}_{i} \boldsymbol{v}_{i}^{\dagger} \\
1 \leq i \leq K-1 .
\end{gathered}
$$

(Note that (72b) is simply (71) with $\boldsymbol{R}=\boldsymbol{I}$ and $\omega_{0}=0$.) Clearly, such a matrix is lossless. Furthermore, its determinant has the form $c \Pi_{i=1}^{K-1}\left(-a_{i}^{*}+z^{-1}\right) /\left(1-a_{i} z^{-1}\right)$, (with $|c|=1$ ) showing that its degree is $K-1$. In this way, nontrivial examples of lossless IIR systems of degree $K-1$ can be obtained. However, it is not clear if such a representation is sufficiently general. In this section, we will show that any IIR lossless matrix of degree $K-1$ can be expressed as a product of the form (72a) and a unitary matrix.

Let us first formally define the problem: Given an $M \times$ $M$, degree $K-1$ IIR lossless matrix $\boldsymbol{H}_{K-1}(z)$ with poles $z_{i}, 1 \leq i \leq K-1$, we wish to write

$$
\boldsymbol{H}_{K-1}(z)=\boldsymbol{V}_{1}(z) \boldsymbol{H}_{K-z}(z)
$$

where $V_{1}(z)$ is as in (72b) and $H_{K-2}(z)$ is an $M \times M$ IIR lossless matrix of degree $K-2$. We also require the set of poles of $\boldsymbol{H}_{K-2}(z)$ to be contained in that of $\boldsymbol{H}_{K-1}(z)$. Once accomplished, this will constitute the basic step of the synthesis procedure which can be repeated a sufficient number of times until a factorization of $\boldsymbol{H}_{K-1}(z)$ in terms of degree-one IIR sections is obtained.

Our task now is to give an assignment rule for $\boldsymbol{v}_{1}$ and $a_{1}$ such that $\boldsymbol{H}_{K-2}(z)$ is indeed lossless and of degree $K$ -2 . Before we do so, recall that det $\boldsymbol{H}_{K-1}(z)$ has the form

$$
\operatorname{det} \boldsymbol{H}_{K-1}(z)=e^{i \theta} \prod_{i=1}^{K-1} \frac{-z_{i}^{*}+z^{-1}}{1-z_{i} z^{-1}} .
$$

Note that $1 / z_{i}^{*}, 1 \leq i \leq K-1$ are the determinantal zeros of $\boldsymbol{H}_{K-1}(z)$ [27]. Therefore there exist unit-norm vectors $\boldsymbol{u}_{i}$ such that

$$
\boldsymbol{u}_{i}^{\dagger} \boldsymbol{H}_{K-1}\left(\frac{1}{z_{i}^{*}}\right)=\mathbf{0}, \quad 1 \leq i \leq K-1 .
$$

This observation will be of use later in the synthesis procedure. 
We now propose the following assignment: Let $a_{1}=z_{1}$ and choose $v_{1}$ such that

$$
\boldsymbol{v}_{i}^{\dagger} \boldsymbol{H}_{K-1}\left(\frac{1}{z_{i}^{*}}\right)=\mathbf{0} \text {. }
$$

With these, $\boldsymbol{H}_{K-2}(z)$ becomes

$$
\begin{aligned}
\boldsymbol{H}_{K-2}(z) & =\tilde{\boldsymbol{V}}_{1}(z) \boldsymbol{H}_{K-1}(z) \\
& =\left[\boldsymbol{I}-\boldsymbol{v}_{1} \boldsymbol{v}_{1}^{\dagger}+\frac{-z_{1}+z}{1-z_{1}^{*} z} \boldsymbol{v}_{1} \boldsymbol{v}_{1}^{\dagger}\right] \boldsymbol{H}_{K-1}(z) .
\end{aligned}
$$

Observe that since both $\boldsymbol{H}_{K-1}(z)$ and $\boldsymbol{V}_{1}(z)$ are paraunitary, $\boldsymbol{H}_{K-2}(z)$ is guaranteed to be paraunitary by construction.

We will address the stability issue next. $\boldsymbol{H}_{K-2}(z)$, given by (77) seems to have a pole at $1 / z_{1}^{*}$. Since $\left|1 / z_{1}^{*}\right|>$ 1 , such a pole would cause $\boldsymbol{H}_{K-2}(z)$ to be unstable. We claim that this apparent pole is automatically cancelled by the above choice of $\boldsymbol{v}_{1}$. To see this, observe that since $\boldsymbol{H}_{K-1}(z)$ is analytic outside the unit circle, it can be expanded into a Taylor series around $z=1 / z_{1}^{*}$, i.e., it can be written as

$$
\begin{aligned}
\boldsymbol{H}_{K-1}(z)= & \boldsymbol{P}+\left(z-\frac{1}{z_{1}^{*}}\right) \boldsymbol{Q} \\
& +\frac{1}{2}\left(z-\frac{1}{z_{1}^{*}}\right)^{2} \boldsymbol{R}+\cdots
\end{aligned}
$$

where

$$
\boldsymbol{P}=\boldsymbol{H}_{K-1}\left(\frac{1}{z_{1}^{*}}\right), \quad \boldsymbol{Q}=\left.\left(\frac{\partial}{\partial z}\right) \boldsymbol{H}_{K-1}(z)\right|_{z=1 / z_{1}^{*}}
$$

and

$$
\boldsymbol{R}=\left.\left(\frac{\partial^{2}}{\partial z^{2}}\right) \boldsymbol{H}_{K-1}(z)\right|_{z=1 / / i^{*}}
$$

If we substitute (78) in (77), we obtain

$$
\begin{aligned}
\boldsymbol{H}_{K-2}(z)= & {\left[\boldsymbol{I}-\left(1-\frac{-z_{1}+z}{1-z_{1}^{*} z}\right) \boldsymbol{v}_{1} \boldsymbol{v}_{1}^{\dagger}\right] } \\
& \cdot\left[\boldsymbol{P}+\left(z-\frac{1}{z_{1}^{*}}\right)\right. \\
& \left.\cdot Q+\frac{1}{2}\left(z-\frac{1}{z_{1}^{*}}\right)^{2} \boldsymbol{R}+\cdots\right]
\end{aligned}
$$

or, after some arrangement

$$
\begin{aligned}
\boldsymbol{H}_{K-2}(z)= & \boldsymbol{H}_{K-1}(z)+\alpha\left(z, z_{1}\right) \\
& \cdot \boldsymbol{v}_{1}\left[\boldsymbol{v}_{1}^{\dagger} \boldsymbol{P}+\left(z-\frac{1}{z_{1}^{*}}\right) \boldsymbol{v}_{1}^{\dagger} \boldsymbol{Q}\right. \\
& \left.+\frac{1}{2}\left(z-\frac{1}{z_{1}^{*}}\right)^{2} \boldsymbol{v}_{1}^{\dagger} \boldsymbol{R}+\cdots\right]
\end{aligned}
$$

where $\alpha\left(z, z_{1}\right)=\left(1 / z_{1}^{*}\right)\left(\left(1+z_{1}\right)-z\left(1+z_{1}^{*}\right)\right) /$ $\left(z-\left(1 / z_{1}^{*}\right)\right)$. It is clear from $(80)$ that the only problem causing term is $\alpha\left(z, z_{1}\right) \boldsymbol{v}_{1} \boldsymbol{v}_{1}^{\dagger} \boldsymbol{P}$. Recall, however that $\boldsymbol{P}$ $=\boldsymbol{H}_{K-1}\left(1 / z_{1}^{*}\right)$ and that $\boldsymbol{v}_{1}$ was chosen to satisfy $(76)$. With these, (80) simplifies to

$$
\begin{aligned}
\boldsymbol{H}_{K-2}(z)= & \boldsymbol{H}_{K-1}(z)+\alpha\left(z, z_{1}\right)\left(z-\frac{1}{z_{1}^{*}}\right) \\
& \cdot\left[\boldsymbol{v}_{1} \boldsymbol{v}_{1}^{\dagger} \boldsymbol{Q}+\frac{1}{2}\left(z-\frac{1}{z_{1}^{*}}\right) \boldsymbol{v}_{1} \boldsymbol{v}_{1}^{\dagger} \boldsymbol{R}+\cdots\right]
\end{aligned}
$$

which is analytic at $z=1 / z_{1}^{*}$.

Having thus established stability of $\boldsymbol{H}_{K-2}(z)$, we next address the issue of degree reduction. If we take the determinant of both sides of (73), we obtain

$$
\operatorname{det} \boldsymbol{H}_{K-1}(z)=\frac{-z_{1}^{*}+z^{-1}}{1-z_{1} z^{-1}} \operatorname{det} \boldsymbol{H}_{K-2}(z) .
$$

Since there are no cancellations on the right-hand side of (82), we can write

$$
\text { deg det } \boldsymbol{H}_{K-1}(z)=1+\operatorname{deg} \operatorname{det} \boldsymbol{H}_{K-2}(z) .
$$

$\boldsymbol{H}_{K-1}(z)$ and $\boldsymbol{H}_{K-2}(z)$ are both lossless, therefore invoking Property 3 of Section II-B, we can rewrite (83) as

$$
\operatorname{deg} \boldsymbol{H}_{K-1}(z)=1+\operatorname{deg} \boldsymbol{H}_{K-2}(z) .
$$

It follows then that, since $\boldsymbol{H}_{K-1}(z)$ has degree $K-1$, $\boldsymbol{H}_{K-2}(z)$ must have degree $K-2$ as claimed.

Summarizing, given any $M \times M$ IIR lossless matrix $\boldsymbol{H}_{K-1}(z)$ of degree $K-1$ with poles $z_{i}, 1 \leq i \leq K-1$, we can factorize $\boldsymbol{H}_{K-1}(z)$ as in (73), where $\boldsymbol{H}_{K-2}(z)$ is another $M \times M$ IIR lossless matrix of degree $K-2$, by choosing a unit-norm vector $v_{1}$ that satisfies (76). Repeated application of this result gives us a way of expressing a general lossless matrix of degree $K-1$ as a product of degree-one lossless matrices, i.e., enables us to write

$$
\boldsymbol{H}_{\boldsymbol{K}-1}(z)=\boldsymbol{V}_{1}(z) \boldsymbol{V}_{2}(z) \cdots \boldsymbol{V}_{\boldsymbol{K}-1}(z) \boldsymbol{H}_{0}
$$

where $\boldsymbol{H}_{0}$ is a unitary matrix and $\boldsymbol{V}_{i}(z)$ are given by $(72 \mathrm{~b})$. In $(72 b), v_{i}$ are unit-norm vectors chosen such that $\boldsymbol{v}_{i}^{\dagger} \boldsymbol{H}_{K-1-i}\left(1 / z_{i}^{*}\right)=0$. The corresponding structural implementation is shown in Fig. 6.

\section{A Second Synthesis Procedure for Power Complementary IIR Vectors}

Clearly a product of matrices of the form (72a) postmultiplied by a constant unit-norm vector represents a PC IIR vector. To demonstrate that this is a general form for such vectors, we need to show that any (stable) PC IIR vector can be synthesized as such a cascade. The synthesis procedure described in Section IV-B can easily be modified for PC IIR vectors. Consider an $M \times 1$ PC IIR vector $\boldsymbol{H}_{K-1}(z)$ of degree $K-1$ given by (50). Assume as before the polynomials $P_{K-1}^{(j)}(z), 0 \leq j \leq M-1$ and $d_{K-1}(z)$ do not have any common factors. 


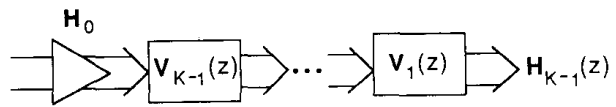

(a)

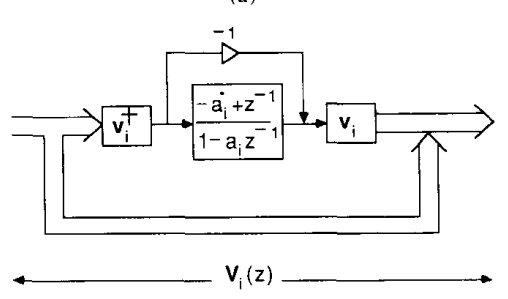

(b)

Fig. 6. (a) The structural implementation of Section IV-B. (b) Internal de tails of $V_{i}(z)$.

Now suppose that we want to synthesize $\boldsymbol{H}_{K-1}(z)$ as a cascade of matrices of the form (72b) terminated by a unitnorm constant vector. An an initial step, we want to write

$$
\boldsymbol{H}_{K-1}(z)=\boldsymbol{V}_{1}(z) \boldsymbol{H}_{K-2}(z)
$$

$(86 a)$

where $\boldsymbol{H}_{K-2}(z)$ has the form

$$
\begin{aligned}
\boldsymbol{H}_{K-2}(z)= & \left(P_{K-2}^{(0)}(z) \quad P_{K-2}^{(1)}(z) \cdots\right. \\
& \left.P_{K-2}^{(M-1)}(z)\right)^{T} / d_{K-2}(z) \\
P_{K-2}^{(i)}(z)= & \sum_{i=0}^{K-2} p_{K-2 . i}^{(j)} z^{-i}, \quad 0 \leq j \leq M-1 \\
d_{K-2}(z)= & \frac{d_{K-1}(z)}{1-z_{1} z^{-1}}
\end{aligned}
$$

and $V_{1}(z)$ is as in (72b). For this, we define the FIR vector $N_{K-1}(z) \triangleq d_{K-1}(z) \boldsymbol{H}_{K-1}(z)$, and propose to let $a_{1}$ $=z_{1}$ and to choose $v_{1}$ such that

$$
v_{1}^{\dagger} N_{K-1}\left(\frac{1}{z_{1}^{*}}\right)=0 \text {. }
$$

Recall that we made a similar choice for $v_{1}$ in the lossless matrix case. In this case, however, we can be more specific and give a closed-form expression for $\boldsymbol{v}_{1}$. Furthermore, it can be shown that this choise is unique. We see from (86a) that, $\boldsymbol{H}_{K-2}(z)$ can be written as

$$
\begin{aligned}
\boldsymbol{H}_{K-2}(z)= & \tilde{\boldsymbol{V}}_{1}(z) \boldsymbol{H}_{K-1}(z) \\
= & {\left[\boldsymbol{I}-\boldsymbol{v}_{1} \boldsymbol{v}_{1}^{\dagger}\right] \boldsymbol{H}_{K-1}(z) } \\
& +\frac{-z_{1}+z}{1-z_{1}^{*} z} \boldsymbol{v}_{1} \boldsymbol{v}_{1}^{\dagger} \boldsymbol{H}_{K-1}(z) .
\end{aligned}
$$

Let us consider the second term on the right-hand side of (88b) first. It follows from (87) that $v_{1}^{\dagger} N_{K-1}(z)=(1-$ $\left.z_{1}^{*} z\right) \lambda(z)$ where the order of $\lambda(z)$ is strictly less than that of the higher order polynomial in $N_{K-1}(z)$. With this, the second term of $(88 \mathrm{~b})$ can be written as

$$
\frac{-z_{1}+z}{1-z_{1}^{*} z} \boldsymbol{v}_{1} \boldsymbol{v}_{1}^{+} \boldsymbol{H}_{K-1}(z)=\frac{\lambda(z) \boldsymbol{v}_{1}}{d_{K-2}(z)}
$$

which is analytic at $z=1 / z_{1}^{*}$. Note that in (89), the order of the denominator polynomial and the maximum order of the numerator polynomials are both reduced.

Let us now consider the first term of (88b). In order to cancel the pole at $z_{1}$, we must have

$$
\left[\boldsymbol{I}-\boldsymbol{v}_{1} \boldsymbol{v}_{1}^{\dagger}\right] \boldsymbol{N}_{K-1}\left(z_{1}\right)=\mathbf{0} .
$$

Note that $\left[\boldsymbol{I}-\boldsymbol{v}_{1} \boldsymbol{v}_{1}^{\dagger}\right]$ has rank $M-1$. Therefore there is a unique vector $\boldsymbol{u}$ (except for a scale factor) such that [ $\boldsymbol{I}$ $\left.-\boldsymbol{v}_{1} \boldsymbol{v}_{1}^{\dagger}\right] \boldsymbol{u}=\mathbf{0}$. By inspection, $\boldsymbol{u}=\boldsymbol{v}_{1}$ works. In view of this, (90) implies that the unique choice for $v_{1}$ is

$$
v_{1} \triangleq \frac{N_{K-1}\left(z_{1}\right)}{\left|N_{K-1}\left(z_{1}\right)\right|} \text {. }
$$

Note that (91) agrees with (87) since

$$
N_{K-1}^{\dagger}\left(z_{1}\right) N_{K-1}\left(\frac{1}{z_{1}^{*}}\right)=0
$$

due to the paraunitary property of $\boldsymbol{H}_{K-1}(z)$. With this choice of $v_{1}$, the first term on the right-hand side of $(88 \mathrm{~b})$ becomes

$$
\begin{aligned}
{\left[\boldsymbol{I}-\boldsymbol{v}_{1} \boldsymbol{v}_{1}^{\dagger}\right] \frac{\boldsymbol{N}_{K-1}(z)}{d_{K-1}(z)} } & =\frac{\left(1-z_{1} z^{-1}\right) \boldsymbol{a}(z)}{d_{K-1}(z)} \\
& =\frac{\boldsymbol{a}(z)}{d_{K-2}(z)}
\end{aligned}
$$

where again the order of the denominator polynomial and the maximum order of the numerator polynomials are both reduced.

Putting these results together, we conclude that $H_{K-1}(z)=N_{K-1}(z) / d_{K-1}(z)$ can indeed be factorized as in (86) into a lossless matrix $V_{1}(z)$ and a reduced degree PC IIR vector $\boldsymbol{H}_{K-2}(z)$ by appropriately choosing $\boldsymbol{v}_{1}$. The vector $\boldsymbol{H}_{K-2}(z)$ can in turn be expressed as $\boldsymbol{H}_{K-2}(z)=\boldsymbol{N}_{K-2}(z) / d_{K-2}(z)$. Repeatedly applying the described step, we can synthesize $\boldsymbol{H}_{K-1}(z)$ as the cascade

$$
\boldsymbol{H}_{K-1}(z)=\boldsymbol{V}_{1}(z) \boldsymbol{V}_{2}(z) \cdots \boldsymbol{V}_{K-1}(z) \boldsymbol{H}_{0}
$$

where $\boldsymbol{H}_{0}$ is a unit-norm vector, $\boldsymbol{V}_{i}(z)$ are as described by $(72 b)$ with

$$
\boldsymbol{v}_{i}=\frac{\boldsymbol{N}_{K-1-i}\left(z_{i}\right)}{\left|\boldsymbol{N}_{K-1-i}\left(z_{i}\right)\right|}, \quad 1 \leq i \leq K-1
$$

and $z_{i}$ is the $i$ th pole of $\boldsymbol{H}_{K-1}(z)$.

\section{A Synthesis Procedure for $M \times M I I R L B R$ Matrices}

If the matrix $\boldsymbol{H}_{K-1}(z)$ is LBR, then the poles are either real, or occur in complex conjugate pairs, which can be characterized by two real numbers. This suggests the possiblity of obtaining a synthesis procedure (hence, a representation) for IIR LBR matrices in terms of degree-one and degree-two lossless matrices with real coefficients corresponding to real and complex conjugate pole pairs, respectively. Such a synthesis procedure will be outlined in this section for IIR LBR matrices. The procedure can 
be straightforwardly extended to the synthesis of IIR LBR vectors.

Let us first consider the real pole case. Let $\boldsymbol{H}_{K-1}(z)$ be an IIR LBR matrix with a real pole $\alpha$. It follows from Section IV-B that $v$ must be chosen such that $\boldsymbol{v}^{\dagger} \boldsymbol{H}_{K-1}(1 / \alpha)=\mathbf{0}$. Since $\boldsymbol{H}_{K-1}(1 / \alpha)$ is a real matrix, $v$ turns out to be real and therefore the extracted degreeone factor

$$
\boldsymbol{G}(z)=\boldsymbol{I}-\boldsymbol{v} \boldsymbol{v}^{T}+\frac{-\alpha+z^{-1}}{1-\alpha z^{-1}} \boldsymbol{v} \boldsymbol{v}^{T}
$$

is also real for real $z$.

Let us now consider the complex conjugate poles case. For this case, for our later convenience, we will adopt a slightly more general building block which is

$$
\boldsymbol{G}_{i}(z)=\boldsymbol{I}-\boldsymbol{v}_{i} \boldsymbol{v}_{i}^{\dagger}-\frac{1+a_{i}}{1+a_{i}^{*}} \frac{-a_{i}^{*}+z^{-1}}{1-a_{i} z^{-1}} \boldsymbol{v}_{i} \boldsymbol{v}_{i}^{\dagger}
$$

The added factor $-\left(1+a_{i}\right) /\left(1+a_{i}^{*}\right)$ will be used to make the overall degree-two matrix real for real $z$. Note that (96) fits the most general form described by (71) with $e^{i \omega^{(i)}}=-\left(1+a_{i}\right) /\left(1+a_{i}^{*}\right)$. It can easily be verified that this does not change the choice of $v_{i}$ and $a_{i}$ used in the synthesis procedure described in Section IV-B.

Consider an IIR LBR matrix $\boldsymbol{H}_{K-1}(z)$ with the complex conjugate pole pair $\left(z_{i}, z_{i}^{*}\right)$. (The following development is analogous to the one repoted in [15, ch. 11] for the case of continuous-time real-coefficient systems.) As before, let $a_{i}=z_{i}$ and choose the corresponding unit-norm vector $v_{i}$ such that

$$
\boldsymbol{v}_{i}^{\dagger} \boldsymbol{H}_{K-1}\left(\frac{1}{z_{i}^{*}}\right)=\mathbf{0}
$$

The first degree-one matrix, then, is

$$
\boldsymbol{G}_{\mathbf{l}}(z)=\boldsymbol{I}-\boldsymbol{v}_{i} \boldsymbol{v}_{i}^{\dagger}+\frac{1+z_{i}}{1+z_{i}^{*}}+\frac{-z_{i}^{*}+z^{-1}}{1-z_{i} z^{-1}} \boldsymbol{v}_{i} \boldsymbol{v}_{i}^{\dagger}
$$

and the reduced degree matrix that results is given by

$$
\boldsymbol{H}_{K-2}(z)=\tilde{\boldsymbol{G}}_{1}(z) \boldsymbol{H}_{K-1}(z) .
$$

Now, to extract the pole at $z=z_{i}^{*}$, we have to choose another unit-norm vector $\boldsymbol{u}_{i}$ such that

$$
\boldsymbol{u}_{i}^{\dagger} \boldsymbol{H}_{K-2}\left(\frac{1}{z_{i}}\right)=\mathbf{0}
$$

Using (97), (99), (100) in conjunction with the LBR nature of $\boldsymbol{H}_{K-1}(z)$, it can be shown that the choice

$$
\boldsymbol{u}_{i}=\frac{\boldsymbol{G}_{1}^{+}\left(\frac{1}{z_{i}}\right) \boldsymbol{v}_{i}^{*}}{\left|\boldsymbol{G}_{1}^{\dagger}\left(\frac{1}{z_{i}}\right) \boldsymbol{v}_{i}^{*}\right|}
$$

works. Note that $\boldsymbol{u}_{i} \neq \mathbf{0}$ since that would imply that $\boldsymbol{v}_{i}=$ 0. Let us now consider $G_{i}^{\dagger}\left(1 / z_{i}\right) v_{i}^{*}$. Substituting for
$\boldsymbol{G}_{1}^{\dagger}\left(1 / z_{i}\right)$, using the unit-norm nature of $\boldsymbol{v}_{i}$ and simplifying, we find that $\boldsymbol{u}_{i}$ can be written as

$$
u_{i}=\frac{v_{i}^{*}-\eta^{*} v_{i}}{\sqrt{1-|\eta|^{2}}}
$$

where $\eta$ is given by

$$
\frac{1-\left|z_{i}\right|^{2}}{\left(1-\left|z_{i}\right|^{2}\right)-i 2 \operatorname{Im}\left[z_{i}\right]} \boldsymbol{v}_{i}^{T} \boldsymbol{v}_{i}
$$

The degree-one matrix associated with $z_{i}^{*}$ is now fully specified as

$$
\boldsymbol{G}_{2}(z)=\boldsymbol{I}-\boldsymbol{u}_{i} \boldsymbol{u}_{i}^{\dagger}-\frac{1+z_{i}^{*}}{1+z_{i}} \frac{-z_{i}+z^{-1}}{1-z_{i}^{*} z^{-1}} \boldsymbol{u}_{i} \boldsymbol{u}_{i}^{\dagger}
$$

We now consider the product $G_{1}(z) \mathrm{G}_{2}(z)$. After equating denominators, multiplying out and suitably combining terms, we obtain

$$
\begin{aligned}
\boldsymbol{G}_{c}(z)= & \boldsymbol{G}_{2}(z) \boldsymbol{G}_{1}(z) \\
= & \boldsymbol{I}+\frac{2\left(1-z_{i}\right)\left(1-z_{i}^{*}\right)}{\left(1-\left|z_{i}\right|^{2}\right)\left[1-\left|\boldsymbol{v}^{T} \boldsymbol{v}\right|^{2}+\frac{4 \operatorname{Im}\left[z_{i}\right]^{2}}{\left(1-\left|z_{i}\right|^{2}\right)^{2}}\right]} \\
& \cdot \frac{\operatorname{Re}\left[\boldsymbol{b}_{i}\right]+z^{-1} \operatorname{Re}\left[-z_{i}^{*} b_{i}\right]}{1-z^{-1} 2 \operatorname{Re}\left[z_{i}\right]+z^{-2}\left|z_{i}\right|^{2}} \quad(104 \mathrm{a})
\end{aligned}
$$

where

$$
\boldsymbol{b}_{i}=\left(1+z_{i}\right) \boldsymbol{v}_{i}^{*} \boldsymbol{u}_{i}^{\dagger}
$$

The term in brackets in the denominator of (104a) cannot be zero since it can easily be shown that it would lead to $\left\|\boldsymbol{u}_{i}\right\|^{2}=1-|\eta|^{2}=0$ which is a contradiction. Hence (104) is well defined, and all the coefficients are real as claimed. Details in the derivation of (104) are omitted, as they can easily be carried out.

\section{E. The Link to Another General Representation}

In [9], some parametrizations of FIR LBR matrices in terms of planar rotations were derived. These parametrizations were shown to lead to general lattice structures for FIR LBR matrices. The approach used in [9] to parametrize FIR LBR matrices can be generalized for IIR lossless matrices. If the details are carried out, it can be shown that an $M \times M$ IIR lossless matrix $\boldsymbol{H}_{K-1}(z)$ of degree $K-1$ can be implemented by the structure shown in Fig. 7. Each crisscross in Fig. 7(b) has the internal details shown in Fig. 4(c). The characteristics of lossless matrices by these structures is in terms of planar rotations which involve angles, as opposed to the characterization of the structure of Section IV-B which is basically in terms of unit-norm vectors. The presence of angles makes it necessary to compute several cosines and sines especially in applications which require optimization of parameters. 


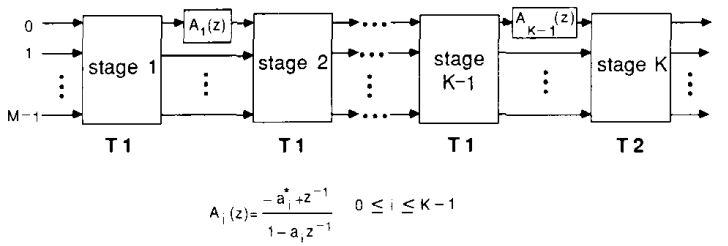

(a)

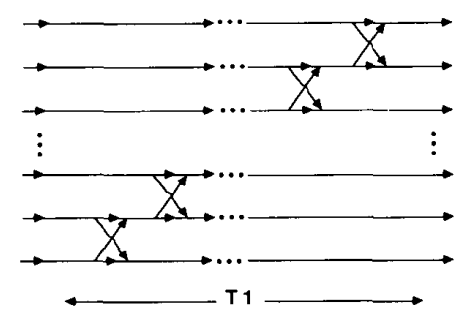

(b)

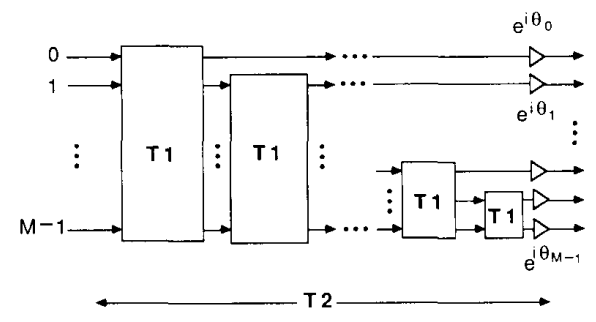

(c)

Fig. 7. (a) Another general lossless IIR structure. (b) Internal details of T1. (c) Internal details of $\mathrm{T} 2$

On most general-purpose computers, the computation of a cosine (sine) is about 20 times slower than a multiplication operation. This makes the structure of Section IV-B more desirable in applications that involve optimization of the parameters. Still, it is of interest to know how the general structure of Fig. 7 is related to the one described in Section IV-B. This will be considered next.

Let us consider the building block $V_{i}(z)$ described by (72b). Given $\boldsymbol{v}_{i}$, we can use Gram-Schmidt orthogonalization procedure [17] to generate the set of vectors $\boldsymbol{v}_{i}, \boldsymbol{u}_{1}$, $u_{2}, \cdots, u_{M-1}$ satisfying

$$
\begin{aligned}
& \boldsymbol{u}_{k}^{\dagger} \boldsymbol{u}_{j}= \begin{cases}1 & k=j \\
0 & \text { otherwise }\end{cases} \\
& \boldsymbol{v}_{i}^{\dagger} \boldsymbol{u}_{j}=0, \quad 1 \leq k, j \leq M-1 .
\end{aligned}
$$

Note that

$$
\boldsymbol{V}_{i}(z) \boldsymbol{v}_{i}=\frac{-a_{i}^{*}+z^{-1}}{1-a_{i} z^{-1}} \boldsymbol{v}_{i}
$$

and

$$
\boldsymbol{V}_{i}(z) \boldsymbol{u}_{j}=\boldsymbol{u}_{j} \quad 1 \leq j \leq M-1 .
$$

Hence $V_{i}(z)$ can be expressed as

$$
\boldsymbol{V}_{i}(z)=\boldsymbol{U}_{i} \boldsymbol{\Lambda}_{i}(z) \boldsymbol{U}_{i}^{\dagger}
$$

where $U_{i}=\left(v_{i} v_{1} \cdots u_{M-1}\right)$ is a unitary matrix and $\boldsymbol{\Lambda}_{i}(z)$ is given by

$$
\boldsymbol{\Lambda}_{i}(z)=\left(\begin{array}{ll}
\frac{-a_{i}^{*}+z^{-1}}{1-a_{i} z^{-1}} & 0 \\
\mathbf{0} & \boldsymbol{I}_{M-1}
\end{array}\right) .
$$

If we substitute (107a) in (85) for $1 \leq i \leq K-1$ and simplify, we obtain

$$
\boldsymbol{H}_{K-1}(z)=\boldsymbol{L}_{1} \boldsymbol{\Lambda}_{1}(z) \boldsymbol{L}_{2} \cdots \boldsymbol{L}_{K-1} \boldsymbol{\Lambda}_{K-1}(z) \boldsymbol{L}_{K}
$$

where $\boldsymbol{L}_{i}$ are unitary matrices. It is possible to represent an $M \times M$ general unitary matrix in terms of $\left(\begin{array}{c}M \\ 2\end{array}\right)$ complex planar rotations and $M$ complex multipliers [18] as shown in Fig. 7(c) (each crisscross in Fig. 7(c) represents a complex planar rotation). Hence, (108) can be depicted as in Fig. 7(a) with each stage having the internal details of Fig. 7(c). Note that the $M$ complex multipliers of $\boldsymbol{L}_{1}$ can be moved to the right of $\boldsymbol{A}_{1}(z)$ without altering the inputoutput relationship. Also, since the all-pass block $A_{1}(z)$ affects only the topmost line, the $\left(M_{2}^{M-1}\right)$ complex crisscrosses of $L_{1}$ that do not touch this line can be moved to the right and coalesced with $L_{2}$ to form a new unitary matrix. Having done so, we are left with $M-1$ complex planar rotations (crosscrosses) in the first stage. The newly formed unitary matrix of the second stage can be redecomposed as shown in Fig. 7(c). We can then once again move the $M$ multipliers and $\left({ }^{M-1}\right)$ crisscrosses to the right and merge them with $L_{3}$. If this process is repeated, then the first $\boldsymbol{K}-1$ stages have $M-1$ complex planar rotations and the last stage remains a general unitary matrix with $\left(\begin{array}{c}M \\ 2\end{array}\right)$ complex planar rotations and $M$ complex multipliers. With these, the representation for $M \times M$ IIR lossless matrices described in Section IV-B becomes equivalent to the one depicted in Fig. 7.

\section{Some Properties of the Representation of SECTION IV}

The representation introduced in Section IV-A for IIR lossless matrices has some interesting properties. One of these is that the resulting structural implementations are minimal, i.e., they use the least number of scalar delays possible to implement a given IIR lossless matrix. A second one which is less obvious is that these structures are orthogonal implementations, i.e., the state-space descriptions $(\boldsymbol{A}, \boldsymbol{B}, \boldsymbol{C}, \boldsymbol{D})$ of the structures are such that the matrix $R=\left(\begin{array}{l}A B \\ C\end{array}\right)$ is unitary. The structures proposed in Section IV therefore have all the advantages of orthogonal implementations, elaborated in [21] and [22]. Finally, the representation of Section IV-A is also minimal in the number of parameters used. In this section, we will look at these properties closely.

\section{A. Orthogonality of the Implementation}

We will show that the matrix $R=\left(\begin{array}{c}A B \\ C\end{array}\right)$ corresponding to the state-space description $(\boldsymbol{A}, \boldsymbol{B}, \boldsymbol{C}, \boldsymbol{D})$ of the struc- 
ture described in Section IV-B is unitary, using an energy balance argument.

Let us first consider the building block $V_{i}(z)$ shown in Fig. 8(b), where the state variable is denoted by $x_{i}(n)$. If we denote the input and output matrices corresponding to this block by $\boldsymbol{u}_{i}(n)$ and $\boldsymbol{y}_{i}(n)$, respectively, and the statespace matrix by $\boldsymbol{R}_{i}$, we can write

$$
\left(x_{i}(n+1) \boldsymbol{y}_{i}(n)\right)^{T}=\boldsymbol{R}_{i}\left(x_{i}(n) \boldsymbol{u}_{i}(n)\right)^{T} .
$$

The state-space equations for this block are

$$
\begin{aligned}
x_{i}(n+1)= & a_{i} x_{i}(n)+\sqrt{1-\left|a_{i}\right|^{2}} \boldsymbol{v}_{i}^{\dagger} u_{i}(n) \\
y_{i}(n)= & \sqrt{1-\left|a_{i}\right|^{2}} \boldsymbol{v}_{i} x_{i}(n) \\
& +\left[I-\left(1+a_{i}^{*}\right) \boldsymbol{v}_{i} \boldsymbol{v}_{i}^{\dagger}\right] \boldsymbol{u}_{i}(n) .
\end{aligned}
$$

Therefore, $\boldsymbol{R}_{i}$ is given by

$$
\boldsymbol{R}_{i}=\left(\begin{array}{lc}
a_{i} & \sqrt{1-\left|a_{i}\right|^{2}} \boldsymbol{v}_{i}^{\dagger} \\
\sqrt{1-\left|a_{i}\right|^{2}} \boldsymbol{v}_{i} & {\left[\boldsymbol{I}-\left(1+a_{i}^{*}\right) \boldsymbol{v}_{i} \boldsymbol{v}_{i}^{\dagger}\right]}
\end{array}\right)
$$

and can easily be verified to be unitary using the fact that $\boldsymbol{v}_{i}$ is unit norm. It follows from the unitariness of $\boldsymbol{R}_{i}$ and (109) that

$$
\left|x_{i}(n+1)\right|^{2}+\left\|y_{i}(n)\right\|^{2}=\left|x_{i}(n)\right|^{2}+\left\|\boldsymbol{u}_{i}(n)\right\|^{2}
$$

for $V_{i}(z)$

Let us now consider the structure of Fig. 8(a), with internal details as in Fig. 8(b). Using (112), we can write

$$
\begin{aligned}
\left|x_{1}(n+1)\right|^{2}+\left\|\boldsymbol{y}_{1}(n)\right\|^{2}= & \left|x_{1}(n)\right|^{2} \\
& +\left\|\boldsymbol{H}_{0} \boldsymbol{u}(n)\right\|^{2} \\
\left|x_{2}(n+1)\right|^{2}+\left\|\boldsymbol{y}_{2}(n)\right\|^{2}= & \left|x_{2}(n)\right|^{2} \\
& +\left\|\boldsymbol{y}_{1}(n)\right\|^{2} \\
& \vdots \\
\left|x_{K-1}(n+1)\right|^{2}+\left\|\boldsymbol{y}_{K-1}(n)\right\|^{2}= & \left|x_{K-1}(n)\right|^{2} \\
& +\left\|\boldsymbol{y}_{K-2}(n)\right\|^{2} .
\end{aligned}
$$

If we add both sides of the equalities in (113) and make the necessary cancellations, we obtain

$$
\begin{gathered}
\sum_{i=1}^{K-1}\left|x_{i}(n+1)\right|^{2}+\left\|\boldsymbol{y}_{i}(n)\right\|^{2} \\
=\sum_{i=1}^{K-1}\left|x_{i}(n)\right|^{2}+\|\boldsymbol{u}(n)\|^{2}
\end{gathered}
$$

where we have made use of the facts that $\left\|\boldsymbol{y}_{K-1}(n)\right\|^{2}=$ $\|\boldsymbol{y}(n)\|^{2}$, and $\left\|\boldsymbol{H}_{0} \boldsymbol{u}(n)\right\|^{2}=\|\boldsymbol{u}(n)\|^{2}$. Therefore, if we

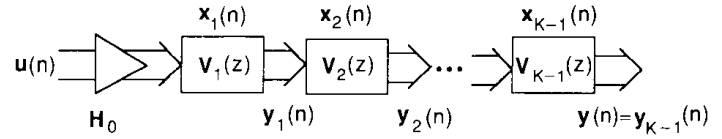

(a)

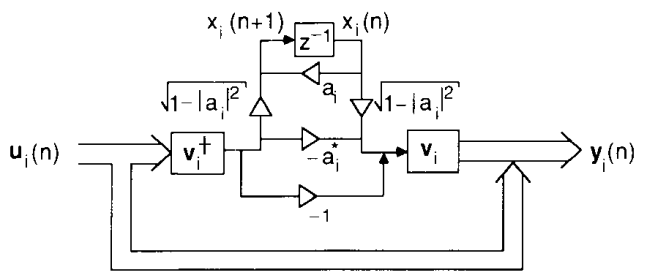

(b)

Fig. 8. (a) The IIR lossless structure. (b) Internal details of $V_{i}(z)$.

let $\boldsymbol{a}=(x(n) \boldsymbol{u}(n))^{T}$, we can rewrite (114) as

$$
\boldsymbol{a}^{\dagger} \boldsymbol{R}^{\dagger} \boldsymbol{R} \boldsymbol{a}=\boldsymbol{a}^{\dagger} \boldsymbol{a} \quad \forall \boldsymbol{a} .
$$

It can easily be shown that (115) implies that $\boldsymbol{R}=\boldsymbol{I}$, thus proving that the general IIR lossless structure of Section IV-B (also shown in Fig. 8) is an orthogonal implementation.

Note that the same proof holds for the case when $\boldsymbol{H}_{0}$ is a unit-norm vector instead of a unitary matrix. Hence the structure of Section IV-C for IIR lossless vectors is also orthogonal. A similar energy balance argument can be used to show that the structures of Section III are also orthogonal implementations.

\section{B. Minimality of the Number of Parameters}

In this section, we will calculate the number of degrees of freedom involved in the representation of Section IV, and show this number to be minimum.

Let us first consider the number of degrees of freedom that a matrix has of the form (72b). The unit-norm vector $\boldsymbol{v}_{i}$ has $2 M-1$ degrees of freedom. Suppose that we factor out a common phase term from $\boldsymbol{v}_{i}$ such that one of its entries becomes real. Clearly, this phase term will not appear in the product $\boldsymbol{v}_{i} \boldsymbol{v}_{i}^{\dagger}$, hence it cannot be counted as a freedom. Therefore, $\boldsymbol{v}_{i} \boldsymbol{v}_{i}^{\dagger}$ has $2(M-1)$ degrees of freedom. Also, the complex pole $a_{i}$ (which is subject only to the inequality constraint $\left|a_{i}\right|<1$ ), contributes 2 degrees of freedom. Thus (72b) has a total of $2 M$ degrees of freedom. On the other hand, the $M \times M$ unitary matrix $R$ can be shown [20] to have $\boldsymbol{M}^{2}$ degrees of freedom. Combining these, we conclude that any $M \times M$ lossless matrix $\boldsymbol{H}(z)$ of degree $K-1$ can be represented as in (72b) by

$$
N_{m}=2 M(K-1)+M^{2}
$$

parameters.

Let $N_{s}$ denote the smallest possible number of parameters required to represent such a matrix. Clearly, $N_{s} \leq$ $N_{m}$. To show the equality, all we need to do is to construct an example of an $M \times M$ IIR lossless matrix of degree $K$ - 1 which does have $N_{p}$ degrees of freedom. The construction we will give is an IIR and complex generalization of the one in Section IV of [9]. 
Recall from Section III-C that an $M \times 1$ PC IIR vector $\boldsymbol{h}_{0}(z)$ of degree $K-1$ has $2 M K-1$ degrees of freedom. Such a vector can be implemented by the lattice structure of Section III-B. Therefore we can write

$$
\boldsymbol{h}_{0}(z)=\boldsymbol{S}(z) \boldsymbol{v}_{0}
$$

where $\boldsymbol{S}(z)=\boldsymbol{W}_{1}(z) \boldsymbol{W}_{2}(z) \cdots \boldsymbol{W}_{K-1}(z)$ is an $M \times M$ IIR lossless matrix and $\boldsymbol{v}_{0}$ is a unit-norm vector. Now consider an $M \times M$ unitary matrix $V$ given by

$$
\boldsymbol{V}=\left(\begin{array}{llll}
\boldsymbol{v}_{0} & \boldsymbol{v}_{1} \cdots \boldsymbol{v}_{M-1}
\end{array}\right)
$$

If we define

$$
\boldsymbol{H}(z) \triangleq S(z) \boldsymbol{V}
$$

then clearly $\boldsymbol{H}(z)$ has $\boldsymbol{h}_{0}(z)$ as its first column. Also $\boldsymbol{H}(z)$ is lossless by construction and has degree $K-1$ by Property 3 of Section II-B. Let us now count the degrees of freedom that we could exercise in the construction of such a matrix. Since an arbitrary $M \times M$ unitary matrix has $M^{2}$ degrees of freedom and $\boldsymbol{V}$ has its first column $\boldsymbol{v}_{0}$ already fixed, it has $M^{2}-(2 M-1)$ degrees of freedom left. The total number of degrees of freedom that we could exercise in the construction of $\boldsymbol{H}(z)$ is therefore the sum of the number of degrees of freedom of $\boldsymbol{h}_{0}(z)$ and $\boldsymbol{v}_{1}, \boldsymbol{v}_{2}$, $\cdots, v_{M-1}$. This number is $(2 M K-1)+\left(M^{2}-2 M\right.$ +1 ) which can easily be simplified to $N_{m}$ in (116). This concludes the proof.

Since we have established the existence of an $M \times M$ IIR lossless matrix of degree $K-1$ with $N_{m}$ degrees of freedom, we can write $N_{s}=N_{m}$, which shows the minimality of the structure of Section IV-B in terms of the number of parameters used.

Note also that the structure of Section IV-C for IIR lossless vectors uses $2 M(K-1)+2 M-1$ parameters to implement a general PC IIR vector of degree $K-1$. This number simplifies to $2 \mathrm{MK}-1$ which is the number of degrees of freedom such a vector has. Therefore, the structure of Section IV-C is also minimal.

\section{Concluding Remarks}

The main purpose of this paper has been to obtain general structural representations for IIR PC vectors and lossless square matrices. The generality of these matrices can make them desirable in applications that involve optimization of some parameters. Since the structures cover every PC vector or lossless square matrix, they enable the search for an optimum to be automatically conducted over the set of all such vectors or matrices. It should be stressed, however, that there are computationally more efficient implementation methods (as far as the number of operations are concerned) if the generality of the implementation is not the main concern. Examples of such less general and more efficient implementations can be found in [11] and [12].

\section{APPENDIX}

While investigating the Smith-McMillan form of a lossless matrix $\boldsymbol{H}(z)$ in Section II-C, we saw that the ma- trices $\boldsymbol{\Lambda}_{1}(z)$ and $\boldsymbol{\Lambda}_{2 *}^{-1}(z)$ must coincide except for a possible relabeling of entries. We will now show exactly how this relabeling takes place. From (32), we can write

$$
\epsilon_{0}(z)=a_{l, 1} z^{b_{i,}} \hat{\phi}_{l_{0}}(z)
$$

By (16d)

$$
\hat{\phi}_{l_{0}}(z)=\hat{\phi}_{M-1}(z) \gamma_{0}(z) \cdots \gamma_{M-2-l_{0}}(z) .
$$

If we define $\alpha_{0}(z) \triangleq \gamma_{0}(z) \cdots \gamma_{M-2-1_{0}}(z), \epsilon_{0}(z)$ becomes

$$
\epsilon_{0}(z)=a_{l_{0}} z^{b_{l_{1}}} \hat{\phi}_{M-1}(z) \alpha_{0}(z)
$$

By the same reasoning, we can write

$$
\epsilon_{1}(z)=a_{1} z^{b_{1}} \hat{\phi}_{M-1}(z) \alpha_{1}(z)
$$

where $\alpha_{1}(z)=\gamma_{0}(z) \cdots \gamma_{M-2-l_{1}}(z)$. On the other hand, by $(16 \mathrm{c})$

$$
\epsilon_{1}(z)=\beta(z) \epsilon_{0}(z)
$$

If we substitute (A3) and (A4) in (A5), cancel common terms from both sides and rewrite, we obtain

$$
\beta(z)=c z^{d} \frac{\alpha_{1}(z)}{\alpha_{0}(z)}
$$

where $d$ is an integer and $c$ is a complex constant. Since $\beta(z)$ is a polynomial, the nontrivial polynomial $\alpha_{0}(z)$ must divide $\alpha_{1}(z)$, i.e., we must have $l_{0} \geq l_{1}$. This argument can be used repeatedly in conjunction with the polynomials $\epsilon_{i}(z)$ and $\epsilon_{i+1}(z)$ to show that $l_{i} \geq l_{i+1}$. As a result, we find that $l_{i}$ are related by $l_{0} \geq l_{1} \geq \cdots \geq$ $l_{M-1}$. Since $l_{0} \cdots, l_{M-1}$ represents a permutation of the integers $0, \cdots, M-1$, the only possibility is to have $l$ $=M-1-j$.

\section{REFERENCES}

[1] R. E. Crochiere and L. R. Rabiner, Multirate Digital Signal Processing. Englewood Cliffs, NJ: Prentice-Hall, 1983.

12] M. J. Smith and T. P. Barnwell, III, "A new filter-bank theory for time-frequency representation," IEEE Trans. Acoust., Speech, Signal Processing, vol. ASSP-35, pp. 314-327. Mar. 1987.

[3] M. Vetterli, "A theory of multirate filter banks," IEEE Trans. Acoust., Speech, Signal Processing, vol. ASSP-35, pp. 356-372. Mar. 1987.

[4] R. V. Cox, D. E. Bock, K. B. Bauer, J. D. Johnston, and J. H Snyder, "The analog voice privacy system," AT\&T Tech. J. vol. 66, issue 1, Jan./Feb. 1987.

[5] M. R. Portnoff, "Time-frequency representation of digital filters and systems based on short-time Fourier analysis," IEEE Trans. Acoust., Speech, Signal Processing, vol. ASSP-28, pp. 55-69. Feb. 1980.

[6] P. P. Vaidyanathan and S. K. Mitra, "Low passband-sensitivity filters: A generalized viewpoint and synthesis procedures." Proc. IEEE. vol. 72, pp. 404-423, Apr. 1984

[7] P. P. Vaidyanathan, “Theory and design of $M$-channel maximally decimated quadrature mirror filters with arbitrary $M$, having the perfect-reconstruction property," IEEE Trans. Acoust., Speech, Signal Processing, vol. ASSP-35, pp. 476-492, Apr. 1987.

[8] T. Q. Nguyen and P. P. Vaidyanathan, "Maximally decimated perfect-reconstruction FIR filter banks with pairwise mirror-image analysis (and synthesis) frequency responses," IEEE Trans. Acoust., Speech, Signal Processing, vol. ASSP-36, pp. 693-706, May 1988.

[9] Z. Doğanata, P. P. Vaidyanathan, and T. Q. Nguyen, "General synthesis procedures for FIR lossless transfer matrices, for perfect-reconstruction multirate filter bank applications," IEEE Trans. Acoust., Speech, Signal Processing, vol. ASSP-36, pp. 1561-1574. Oct. 1988. 
[10] P. P. Vaidyanathan, "Passive cascaded-lattice structures for low-sensitivity FIR filter design, with applications to filter banks,"' IEEE Trans. Circuits Syst., vol. CAS-33, pp. 1045-1064, Nov. 1986.

[11] P. P. Vaidyanathan, P. A. Regalia, and S. K. Mitra, "Design of doubly complementary IIR digital filters using a single complex allpass filter, with multirate applications,"' IEEE Trans. Circuits Syst. vol. CAS-34, pp. 378-389, Apr. 1987.

[12] P. P. Vaidyanathan, S. K. Mitra, and Y. Neuvo, "A new approach to the realization of low-sensitivity IIR digital filters," IEEE Trans. Acoust., Speech, Signal Processing, vol. ASSP-34, pp. 350-361, Apr. 1986

[13] P. P. Vaidyanathan, T. Q. Nguyen, Z. Doğanata, and T. Saramaki, "Improved technique for design of perfect-reconstruction FIR QMF banks, with lossless polyphase matrices," IEEE Trans. Acoust. Speech, Signal Processing, vol. ASSP-37, pp. 1042-1056, July 1989

[14] P. P. Vaidyanathan, T. Q. Nguyen, and T. Saramaki, "Improved approach for design of perfect-reconstruction FIR QMF banks, with lossless lattice structures," in Proc. IEEE Int. Conf. ASSP (New York), Apr. 1988, pp. 1471-1474.

[15] V. Belevitch, Classical Network Synthesis. San Francisco, CA. Holden-Day, 1968.

[16] B. D. Anderson and S. Vongpanitlerd, Network Analysis and Synthe sis. Englewood Cliffs, NJ: Prentice-Hall, 1973.

[17] T. Kailath, Linear Systems. Englewood Cliffs, NJ: Prentice-Hall, 1980.

[18] A. V. Oppenheim and R. W. Schafer, Digital Signal Processing Englewood Cliffs, NJ: Prentice-Hall, 1975

[19] J. Franklin, Matrix Theory. Englewood Cliffs, NJ: Prentice-Hall, 1968.

[20] F. D. Murnaghan, The Unitary and Rotation Groups. Washington, D.C.: Spartan, 1962

[21] P. P. Vaidyanathan, "A unified approach to orthogonal digital filters and wave digital filters, based on LBR two-pair extraction," IEEE Trans. Circuits Syst., vol. CAS-32, pp. 673-686, July 1985.

$122]$ P. Dewilde and E. Deprettere, "Orthogonal cascade realization of real multiport digital filters," Int. J. Circuit Theory Appl., vol. 8, pp. $245-277,1980$

[23] F. R. Gantmacher, The Theory of Matrices, vol. I. New York: Chel sea, 1977.

[24] B. McMillan, "Introduction to formal realization theory," Bell Syst. Tech. J., vol. 31, pp. 217-279, 541-600, 1952.

[25] G. D. Forney, Jr., "Minimal beses of rational vector spaces, with applications to multivariable linear systems," SIAM J. Contr., vol. 13, pp. 493-520, 1975

[26] Z. Doğanata and P. P. Vaidyanathan, "New minimal structures for the implementation of power complementary IIR systems," in Proc. IEEE Int. Symp. Circuits Syst. (Portland), May 1989.
[27] P. P. Vaidyanathan and Z. Doğanata, "The role of lossless systems in modern digital signal processing: A tutorial," IEEE Trans. Education, vol. 32, pp. 181-197, Aug. 1989

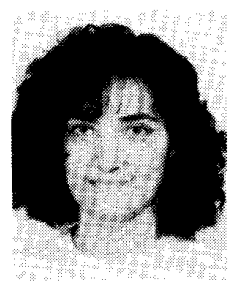

Zinnur Doğanata (S'87-M'88) was born in Ankara, Turkey, on October 29,1959 . She received the B.S. and M.S. degrees in electrical engineering from the Middle East Technical University, Ankara, in 1981 and 1984 , respectively, and the $\mathrm{Ph} . \mathrm{D}$. degree in electrical engineering from the California Institute of Technology, Pasadena, in 1989

During the years 1981-1984, she worked part time for the Military Electronic Industries of Turkey. She is presently with the IBM T. J. Watson Research Center, Yorktown Heights, NY. Her main research interests are in digital signal processing and lossless systems.

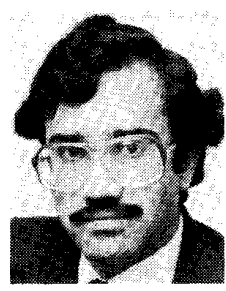

P. P. Vaidyanathan ( $\mathrm{S}^{\prime} 80-\mathrm{M}^{\prime} 83-\mathrm{SM}^{\prime} 88$ ) was born in Calcutta, India, on October 16,1954 . He received the B.Sc. (Hons.) degree in physics, and $B$.Tech. and M.Tech. degrees in radiophysics and electronics from the University of Calcutta, India, in 1974,1977 , and 1979 , respectively, and the $\mathrm{Ph} . \mathrm{D}$. degree in electrical and computer engineering from the University of California, Santa Barbara, in 1982

He was a postdoctoral fellow at the University of California, Santa Barbara, from Septembe 1982 to February 1983. In March 1983, he joined the California Institute of Technology, Pasadena, as an Assistant Professor of Electrical Engineering, and is currently an Associate Professor in the same Department. His main research interests are in digital signal processing, multirate filter bank systems, filter design, adaptive filtering, and multivariable system theory.

Dr. Vaidyanathan served as the Vice-Chairman of the Technical Program Committee for the 1983 IEEE International Symposium on Circuits and Systems and as an Associate Editor for the IEEE TRANSACTIONS ON CIRCUITS AND SYSTEMS, for the period 1985-1987. He was recipient of the award for Excellence in Teaching at the California Institute of Technology, for the year 1983-1984. He is a recipient of NSF's Presidential Young Investigator Award, starting from 1986. In 1989 he received the IEEE ASSP Senior Award for his paper on multirate perfect-reconstruction filter banks In 1990 he was recipient of the S. K. Mitra Memorial Award from the Institute of Electronics and Telecommunications Engineers, India, for his joint paper in the IETE journal. 